\title{
Protocols Design and Area Division for Privacy-preserving Delay-aware Authentication in Vehicular Networks
}

\author{
Qianpeng Wang, Deyun Gao, Senior Member, IEEE, Chuan Heng Foh, Senior Member, IEEE, Hongke Zhang, \\ Senior Member, IEEE, Victor C. M. Leung, Fellow, IEEE
}

\begin{abstract}
The problem of security and privacy in vehicular networks is a vital issue, and it attracts increasing attention to address the security vulnerability of vehicular networks. Authentication solutions are introduced for vehicular networks to ensure that network access is only given to authorized users. Among authentication solutions for vehicular networks, group signature not only offers authentication services, but also provides conditional privacy preservation. However, the current group signature approach for authentication in vehicular networks exhibits time-consuming signature verification and poor scalability. To overcome these shortcomings, we propose a flexible and efficient delay-aware authentication scheme (FEDAS) by utilizing edge computing paradigm. In the proposed architecture, we design the authentication group maintaining mechanism and develop the collaborative CRL management method. Moreover, we propose transition zone to solve the reliable authentication problem in border area of the group. To implement the proposed architecture, we propose a model for calculating the length of local CRL, which establishes the relationship between the size of a sub-area and the length of local CRL. And we also design a method for area division based on the length of local CRL, which provides division principle for our authentication scheme. We conduct extensive simulations to verify the effectiveness of our proposed scheme.
\end{abstract}

Index Terms-Delay-aware authentication, edge computing, certificate revocation list (CRL), group signature, signature verification delay, privacy preservation, area division, vehicular networks.

\section{INTRODUCTION}

$\mathbf{V}$ EHICULAR networks promise efficient information exchange which can greatly improve the driving and riding experiences to users. With vehicular networks, vehicles are capable of communicating with everything around them and subscribe to services for better road safety, traffic management,

Copyright (c) 2015 IEEE. Personal use of this material is permitted. However, permission to use this material for any other purposes must be obtained from the IEEE by sending a request to pubs-permissions @ieee.org.

Qianpeng Wang (e-mail: qianpengwang@bjtu.edu.cn), Deyun Gao (e-mail: gaody@bjtu.edu.cn, Corresponding Author) and Hongke Zhang (e-mail: hkzhang@bjtu.edu.cn) are with the National Engineering Laboratory for Next Generation Internet Interconnection Devices, School of Electronic and Information Engineering, Beijing Jiaotong University, Beijing 100044, China.

Chuan Heng Foh (e-mail: c.fohesurrey.ac.uk) is with 5GIC, Institute for Communication Systems, Department of Electrical and Electronic Engineering, University of Surrey, Surrey GU1 2UX, UK.

Victor C. M. Leung (e-mail: vleung@ece.ubc.ca) is with the Department of Electrical and Computer Engineering, The University of British Columbia, Vancouver, BC V6T 1Z4, Canada. travel comfort, and automatic driving in the future [1]. However, in vehicular networks, vehicles expose themselves to an open wireless environment which is a key security vulnerability [2]. Besides, vehicles carry sensitive user information such as driver's daily activities, and exposing such information may cause severe consequences [3]. It is therefore necessary for vehicular networks to provide adequate security ability before real-world deployment [4]-[6].

In vehicular networks, authentication is an effective means to ensure that network access is given to authorized users. However, authentication also exposes the identity of authorized users to the network which reduces the privacy protection. While privacy-preserving is important, users must also be traceable by traffic management authorities if any misbehavior is detected [7], [8]. Group signature is an authentication technology that not only provides authentication services, but also offers protection of user privacy [9]-[11]. Besides, the group manager can trace and revoke compromised members by distributing Certificate Revocation List (CRL) to all its group members [12]. These characteristics of group signature are suitable for authentication technologies needed for vehicular networks.

However, there are some challenges when exploiting group signature to provide authentication services in vehicular networks. Firstly, the signature verification delay in group signature grows linearly to the length of CRL [13]. This is the time duration needed for the entire signature verification process to complete. When the number of revoked vehicles is large, the signature verification delay will be high, which challenges the proper operation of some delay-sensitive applications in vehicular networks. In addition, the scalability and robustness of the solution are poor since all vehicles in the entire networks are managed by a single centralized group. If the single group manager fails to work, the authentication system in the entire vehicular networks will be paralyzed.

Recently, edge computing has drawn attention from academia and industry to provide timely processing via edge devices [14], [15]. Edge computing is introduced to provide proximal computing services for mobile devices by bringing computational and storage resources to the edge of a network. It offers an open network edge platform and allows thirdparties to exploit the storage and processing capacities, thus bringing innovations and enabling new applications [16]. With edge computing architecture, group management can now be moved from the centralized cloud to the edge of the networks, 
and vehicles can be managed in a distributed way by group managers situated in the network edge, which also makes the fine-grained management of CRL possible.

Motivated by the edge computing paradigm, in this paper, we propose a flexible and efficient delay-aware authentication scheme (FEDAS) to deal with those problems mentioned above. In FEDAS, vehicular networks are divided into multiple small sub-areas where each sub-area is managed by one group manager situated at the edge of the network. Compared with managing the entire area by a single entity, there is fewer revoked vehicles in a smaller sub-area. We propose a collaborative CRL management method in which the global CRL and local CRL are introduced (Section IV-B6). In each sub-area, the shorter local CRL is employed for the verification process. Additionally, groups are isolated from each other, and hence if a group is compromised, the issue is constrained within the group and will not propagate to influence others. However, the group management introduces overhead which may impact the performance. The division of an area should be carefully designed for performance consideration such that we have adequate size of a group to ensure the length of local CRL is short for low delay consideration while avoiding having excessive number of groups to maintain low management overhead. In the following, we summarize the main contributions of this paper:

- We propose a flexible and efficient delay-aware authentication architecture based on edge computing paradigm. Instead of managing the group from the cloud by a single entity, we propose to manage the group in a distributed manner by introducing group managers operating at the edge of a network. This architecture will reduce the verification delay in the authentication process and improve both the scalability as well as robustness of the authentication process.

- Based on the proposed architecture, we develop a CRL management method to make the length of CRL used in the process of verification adjustable, and design the authentication group maintaining mechanism. Additionally, the concept of transition zone is introduced to deal with authentication process at the border of areas where conflict of authentication may occur.

- We propose a model for calculating the length of local CRL, which establishes the relationship between the size of a sub-area and the length of local CRL. And We formulate the area division into an optimization problem and design a heuristic algorithm to solve the problem. The solution provides division principle for our proposed authentication scheme.

The remainder of the paper is organized as follows. In Section II we briefly review the related works. The system model of the proposed FEDAS and security requirements are introduced in Section III. In Section IV, we present the authentication protocol in FEDAS and analyze its security features. The design of area division is then given in Section V. Section VI presents the performances of the proposed scheme. Finally, important conclusions are drawn in Section VII.

\section{RELATED WORKS}

Authentication is a process to validate authorized users such that appropriate access of resources can be granted. Many authentication processes require user identify to be revealed which can lead to privacy issue. In vehicular networks, both authentication and privacy are important issues [17]. Base on traditional Public Key Infrastructure (PKI), pseudonym schemes provide privacy-preserving authentication for vehicular networks by changing pseudonym certificates continuously [18], [19]. For pseudonym schemes, the privacy level of the vehicle is influenced by the frequency of pseudonym change and the number of vehicles that participate in the change simultaneously [20]. Moreover, vehicles need to store a large amount of pseudonym certificates, which will require more storage resource. To revoke a compromised vehicle, all of the pseudonym certificates related to the vehicle must be revoked.

The use of group signature for authentication not only fulfills authentication, but also preserves user privacy by decoupling public verification procedure from the information that would uniquely identify the signer [11]. It is first indicated by Boneh et al. [21] that group signature could be used to provide privacy-preserving authentication in vehicular networks, which then triggers many subsequent works applying and refining group signature technologies for vehicular networks. However, group signature often exhibits lengthy signature verification delay, which is critical for delay-sensitive applications in vehicular networks. To reduce the signature verification delay, batch verification techniques have been proposed [22]-[24]. Batch verification enables vehicle to verify a number of received messages simultaneously instead of each message individually, therefore the overall verification delay is reduced. The verification process consists of two stages: signature check and revocation check. The current solutions reduce delay by only focusing on the stage of signature check but not the stage revocation check which can still be lengthy. In this paper, we reduce the delay of revocation check stage.

Decentralized authentication is proposed to deal with the scalability of group signature by assisting certificate authority to perform authentication and reduce its workload [25][27]. Zhang et al. [25] has proposed a decentralized groupauthentication protocol in which each RSU is used to manage an on-the-fly group within its communication range. The system is scalable because several RSUs are used to share the load of entire system. In [26], [27], the decentralized group model has also been adopted to ease the load of a trusted authority for generating group certificates for On-Board Units (OBUs). However, in these schemes, the number of groups in the entire vehicular network is determined by the number of RSUs, and the size of the group is limited to the coverage of the RSU. A vehicle crossing many small RSUs needs to repeatedly register to each RSU and frequently switch group, which causes performance issue.

Due to the advantages of decentralization, blockchain technologies have been employed to deal with the problems of decentralized authentication in vehicular networks. Lei et al. [28] propose a decentralized key management scheme, in which blockchain structure is used to replace traditional central 
manager in order to decrease the key transfer time and achieve improved robustness. In [29], a blockchain-based distributed framework is proposed to maintain vehicle register information. The information is recorded in a shared ledger, which is immutable and scalable. Dorri et al. [30] propose a blockchainbased distributed solution to share data in the smart vehicle ecosystem which consists of vehicles, manufactures and other service providers. For the solution, only the selected nodes (i.e. cluster heads) participate in managing the blockchain, which makes the system a lightweight solution. In [31], all RSUs in vehicular networks form a blockchain network. The public keys necessary for mutual authentication between vehicle and RSU are stored in blockchain network by the form of smart contracts in a distributed way.

In [32], attribute-based encryption and blockchain technologies are combined to preserve the identity privacy of the vehicle. Blockchain provides the decentralized underlying architecture to store the vehicle public key table where the relationship between blinding identity and public key are recorded. Li et al. [33] develop a zero-knowledge range proof scheme to validate vehicle in a privacy-preserving manner when vehicles are switched between two different blockchains.

In [34], [35], blockchain technologies are used to implement decentralized trust management in vehicular networks. In [34], Yang et al. propose storing the trust values of vehicles in blockchain which is maintained by all RSUs in the network. Both proof-of-work and proof-of-stake are employed jointly to form the consensus mechanisms, and the sum of absolute values of offsets is taken as the stake. Chen et al. [35] leverage blockchain technologies to provide an irreversible trust credits storage mechanism, in which base stations are responsible for managing blockchain and trusted vehicles are selected as the validators to verify transactions.

While blockchain technology has advantages in a decentralized environment, it is challenging to directly apply current blockchain technologies to manage CRL in a distributed way. The operation demands for high decentralization, scalability and attack resistance while maintaining low latency. However, the current consensus algorithms in blockchain fall short on one or more of the demands, which makes the direct application of blockchain to $\mathrm{CRL}$ management challenging [36], [37].

In the literatures mentioned above, blockchain technologies are employed to secure the different aspects of the communication system for vehicular networks. In [29], the central authority for registration information management is replaced by decentralized blockchain nodes to enhance the scalability of the system. In [31], the decentralized structure based on blockchain is used to store the public keys which are employed to secure the communication between vehicle and RSU (V2R). In our scheme, we focus on securing the communication between vehicle and vehicle $(\mathrm{V} 2 \mathrm{~V})$ in a privacy-preserving manner. In [32], a blockchian-assisted scheme is proposed to protect the privacy of the identity of the vehicle. In the scheme, the privacy preservation mechanism requires that the blockchain nodes participate in the authentication process by the way of online. In our scheme, we provide privacy preservation for the identity of the vehicle based on group signature where the third-party is not involved in the authentication process.

In terms of latency, there are two blockchain-based solutions involved. In [32], the public key necessary for message authentication is stored in blockchain which is maintained by several consensus nodes. Every time the vehicle receives a message, it needs to request the public key from the remote blockchain. The request of public key introduces extra communication latency for message authentication. In our proposed scheme, the group public key is shared by all members of the group and the process for message authentication can be completed locally by vehicle itself without extra communication latency. Besides, in [28], blockchain technologies are employed to decrease the key transfer time which is the time taken for delivering the cryptographic materials of the vehicle from a security domain to the neighbor one. In our scheme, we focus on decreasing the verification delay in the process of authentication for $\mathrm{V} 2 \mathrm{~V}$ communications. The focus of our scheme and existing blockchain-based solutions are different. They are not competing technologies, rather they are complementary. Adding blockchain technology to our scheme is possible, but this requires thorough investigation which is out of the scope of this work and more suitable as another standalone research work due to the research involvement.

In the aspect of revocation mechanisms, there are two methods that can be used to deal with compromised group members for group signature: updating keys and distributing CRL. The work in [38] is based on updating keys to revoke the compromised vehicles. The group manager updates the group public key and group secret key for all group members except the revoked members, which prevents the revoked members from accessing the network. Whenever one misbehaving vehicle appears, all vehicles in the network except the misbehaving one are required to update their keys, that is, all vehicles need to register with the group manager again. If there are many compromised vehicles occurring in the short period of time, frequent keys updating will occur, which reduces the lifetime of keys and increases overheads for keys management.

The works presented in [25] and [26] employ the method of distributing CRL to revoked compromised vehicles. In these two schemes, CRL is placed in RSU and thus the process of revocation is performed in RSU. In [25], when a vehicle receives a new message, it sends a query request to RSU and inquires whether the message is sent by a revoked vehicle. This inquiry process introduces additional latency. With constant background messages occurring on the channel, for example Basic Safety Messages are broadcast every 300 milliseconds, any additional message exchange adds extra loads to the network and degrades the overall network performance. In [26], CRL is managed by a centralized authority and is distributed to each RSU periodically. Revocation check is carried out when a vehicle registers to a RSU. If the vehicle requesting to register appears in CRL, the request will be refused.

The main disadvantage of revocation method of distributing CRL is the growing length of CRL which causes lengthy operation and performance degradation. In [39], Lin et al. propose a hybrid operation where both CRL distribution mechanism and key update are employed, and a predefined 
threshold is set to decide which mechanism is used. When the length of CRL is below the threshold, the local revocation mechanism is adopted; otherwise, the mechanism of updating group public key and private key is used.

\section{The System Model And Security Requirements}

In this section, we firstly introduce the system models of FEDAS. Then, we present the security requirements for vehicle to vehicle communications.

\section{A. System Models}

In our proposed authentication architecture, the entire vehicular networks are divided into many blocks where each block is managed individually by a group. Fig. 1 illustrates an example of the proposed architecture. The architecture consists of four entities: global CRL manager, group manager, RSU and vehicles, which are explained below:

- Global CRL Manager: The global CRL manager is responsible for distributing the identity of any revoked vehicle to all group managers.

- Group Manager (GM): GM is the main entity of the group management. As an important entity, GM accomplishes many tasks, including (i) completing vehicle registration and maintaining registration table, (ii) revealing the identity of vehicles performing malicious actions, and (iii) managing local CRL of the group.

- RSU: RSU is the execution unit of the group. RSUs undertake directly the task of group division in the proposed authentication architecture. Multiple adjacent RSUs together constitute a group. RSUs broadcast information about the group periodically. This information includes group identifier, the position information of RSU, zone flag, sequence number of the local CRL, and others. In the system, there are two different types of RSU:

- Non-transition RSU: The non-transition RSU is in the interior of the group. The zone covered by nontransition RSU is called non-transition zone.

- Transition RSU: The transition RSU is situated in the junction of adjacent groups and needs to broadcast group information of these adjacent groups. The zone that is covered by transition RSU is called transition zone.

- Vehicle: Vehicles act as a group member. Signature generation and verification are carried out in vehicles.

With the adaptation of edge computing, we witness an increase number of applications deploying at the network edge [40]. In our proposed solution, RSUs are connected to the edge computing platform where various management tasks are performed. In our proposed solution, the GMs are deployed at the network edge, and the Global CRL Manager is deployed in the cloud on the Internet.

\section{B. Security Requirements}

In this paper, we focus on the security of communication between vehicles. We assume that Global CRL Manager, GMs, and RSUs are protected by good measures, and thus they

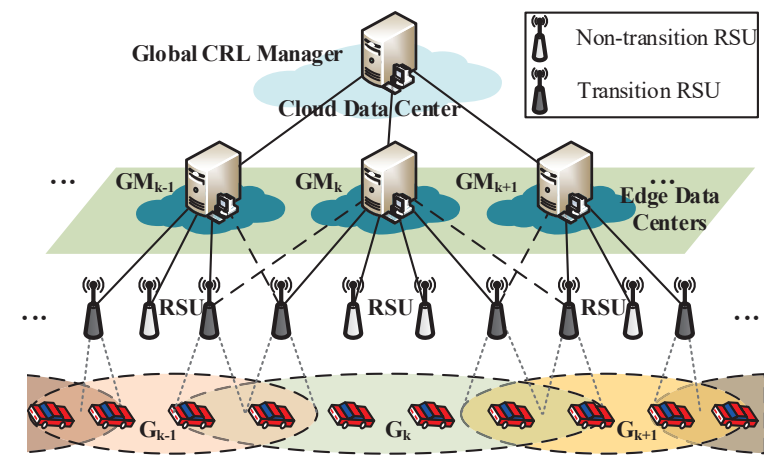

Fig. 1. The architecture of our proposed authentication in the context of edge computing.

are trusted entities. Vehicles communicate with GM securely. To ensure security and privacy protection, only vehicles successfully authenticated by the network are trusted and can communicate securely and anonymously with other trusted entities. However, if malicious actions are detected, the network authority must be able to reveal the identity of the entity performing the malicious actions and have the entity removed from the network to reduce the damage caused by the malicious actions. In this context, the proposed solution must offer entity authentication for authentication, ensure data integrity to achieve integrity in communication, ensure anonymity and message unlinkability for anonymous communication, as well as achieve traceability and membership revocation for network authority to respectively reveal the identity of misbehaved entities and remove misbehaved entities. Besides, attacks such as on-off attack, message spoofing attack, and replay attack should also be resisted. Each of these requirements is elaborated in the following.

1) Entity Authentication: Given a received message, a receiver can check whether the message is produced by an authenticated sender.

2) Data Integrity: Given a received message, a receiver can verify the integrity of the message. In other words, the receiver can always check whether a received message remains unmodified.

3) Anonymity: Given a received message, a receiver is unable to reveal the identify of the sender of the message. The privacy of the sender can thus be protected.

4) Unlinkability: Given two messages, a receiver cannot verify whether the two messages are sent by the same sender.

5) Traceability: If a vehicle is deemed malicious, the GM is able to reveal the identify of the malicious vehicle by inspecting the messages sent from the malicious vehicle.

6) Revocation: If a vehicle is deemed malicious, its membership can be revoked. Once its membership is revoked, messages sent by the malicious vehicle will be considered invalid effectively by all other vehicles.

7) Resistance to On-off Attack: The malicious vehicle may behave badly and attack other vehicles, and then behave well to obtain trust of others again. The legitimate vehicles should be endowed with the ability to prevent this attack.

8) Resistance to Message Spoofing Attack: The malicious vehicle may launch attacks by deliberately broadcasting fake 
messages which may disturb the traffic order. The authentication scheme in vehicular networks should be able to resist this attack.

9) Resistance to Replay Attack: The malicious vehicle may replay messages in the past to cause the receivers to react improperly. Given a received message, a receiver should be able to check whether the message is replayed by a malicious vehicle.

\section{The Flexible and EfFicient Delay-aware AUTHENTICATION SCHEME}

\section{A. Preliminaries}

Our proposed authentication scheme is based on bilinear pairing. The security of the scheme depends on two assumptions, namely the Strong Diffie-Hellman assumption and the Decision Linear assumption. In the following, we briefly cover the bilinear pairing and the two assumptions.

1) Bilinear Pairing [21]: Let $\mathcal{G}_{\mathcal{A}}$ and $\mathcal{G}_{\mathcal{B}}$ be two multiplicative cyclic groups of prime order $p$. Let $g_{1}$ and $g_{2}$ be the generators of $\mathcal{G}_{\mathcal{A}}$ and $\mathcal{G}_{\mathcal{B}}$ respectively, and $\psi$ is a computable isomorphism from $\mathcal{G}_{\mathcal{B}}$ to $\mathcal{G}_{\mathcal{A}}$, with $\psi\left(\mathcal{G}_{\mathcal{B}}\right)=\mathcal{G}_{\mathcal{A}}$. We say that map $e: \mathcal{G}_{\mathcal{A}} \times \mathcal{G}_{\mathcal{B}} \rightarrow \mathcal{G}_{\mathcal{T}}$ is a bilinear pairing if it satisfies (i) computability, where for all $u \in \mathcal{G}_{\mathcal{A}}, v \in \mathcal{G}_{\mathcal{B}}, e(u, v)$ is efficiently computable; (ii) bilinearity, where for all $u \in \mathcal{G}_{\mathcal{A}}$, $v \in \mathcal{G}_{\mathcal{B}}$ and $a, b \in \mathbb{Z}, e\left(u^{a}, v^{b}\right)=e(u, v)^{a b}$; and (iii) nondegeneracy, where $e\left(g_{1}, g_{2}\right) \neq 1$.

2) The Strong Diffie-Hellman Assumption [21]: Let $\mathcal{G}_{\mathcal{A}}$ and $\mathcal{G}_{\mathcal{B}}$ be cyclic groups of prime order $p$, and $g_{1}$ and $g_{2}$ be the generators of $\mathcal{G}_{\mathcal{A}}$ and $\mathcal{G}_{\mathcal{B}}$ respectively. The Strong Diffie-Hellman problem in $\left(\mathcal{G}_{\mathcal{A}}, \mathcal{G}_{\mathcal{B}}\right)$ states as follows: given a tuple $\left(g_{1}, g_{2}, g_{2}^{\gamma}, g_{2}^{\left(\gamma^{2}\right)}, \ldots, g_{2}^{\left(\gamma^{q}\right)}\right)$ as input, output a pair $\left(g_{1}^{1 /(\gamma+x)}, x\right)$ where $x \in \mathbb{Z}_{p}^{*}$. We say that the Strong DiffieHellman assumption holds in $\left(\mathcal{G}_{\mathcal{A}}, \mathcal{G}_{\mathcal{B}}\right)$ if the Strong DiffieHellman problem in $\left(\mathcal{G}_{\mathcal{A}}, \mathcal{G}_{\mathcal{B}}\right)$ is not efficiently computable. With this assumption, two entities can exchange information securely without prior information.

3) The Decision Linear Assumption [21]: Let $\mathcal{G}_{\mathcal{A}}$ be cyclic groups of prime order $p$, and $u, v, h$ are arbitrary generators of $\mathcal{G}_{\mathcal{A}}$. The Decision Linear problem in $\mathcal{G}_{\mathcal{A}}$ is defined as follows: Given $u, v, h, u^{a}, v^{b}, h^{c} \in \mathcal{G}_{\mathcal{A}}$ as inputs, the output is positive if $a+b=c$ and negative otherwise. We say that the Decision Linear assumption holds in $\mathcal{G}_{\mathcal{A}}$ if the Decision Linear problem in $\mathcal{G}_{\mathcal{A}}$ is not efficiently computable. With this assumption, it is computationally hard for an adversary to verify whether two different identities come from the same source.

\section{B. Authentication Protocol in FEDAS}

The role of the FEDAS protocol is to specify various procedures for vehicular communications that can satisfy the earlier mentioned security requirements. In the aspect of network admission or joining, authentication procedure is needed for vehicles to register and join a group in the network. As vehicles travel from a group to another, a procedure to properly maintain group membership is also needed. In the aspect of data communications after network admission, a procedure for registered vehicles to transmit securely and anonymously is required, as well as a procedure for receiving vehicles to verify the integrity of the transmission and the legitimacy of the sender. Finally, to deal with misbehaved vehicles, a procedure to reveal their identity and revoke their membership is required. In the following, we shall detail the procedures for vehicle registration, authentication group maintenance, signature generation for transmission, message verification, identity revealing and tracing, as well as membership revocation via CRL management.

The correctness of our proposed authentication scheme depends on Verifier-Local Revocation (VLR) group signature scheme given in [13]. In our proposed scheme, we focus on developing methods to control the length of CRL without compromising the correctness of the VLR scheme.

1) Vehicle Registration: When vehicle enters a group for the first time, registration to the corresponding GM is required to obtain a certificate. The certificate contains the group public key and the group secret key of the area served by the GM. The group public key in the certificate is $g p k=\left(g_{1}, g_{2}, \omega\right)$ where $g_{2}$ is a generator in $\mathcal{G}_{\mathcal{B}}$ selected uniformly at random, $g_{1}=\psi\left(g_{2}\right)$ and $\psi$ are an computable isomorphism from $\mathcal{G}_{\mathcal{B}}$ to $\mathcal{G}_{\mathcal{A}}, \omega=g_{2}^{\gamma}, \gamma \stackrel{R}{\leftarrow} \mathbb{Z}_{p}^{*}$. The group secret key is $\boldsymbol{g s \boldsymbol { k }}[i]=$ $\left(A_{i}, x_{i}\right)$, where $x_{i} \stackrel{R}{\leftarrow} \mathbb{Z}_{p}^{*}, A_{i} \leftarrow g_{1}^{1 /\left(\gamma+x_{i}\right)}, \gamma+x_{i} \neq 0$, and $i$ is the internal sequence number for the vehicle in the group. Before issuing certificate to vehicle, GM checks the legitimacy of the identity of the vehicle. The identity of a vehicle is unique in vehicular networks, which is signed and issued by the traffic management authority. Only vehicle with a legitimate identity is allowed to obtain certificate from the GM. The GM maintains a registration table, which records the mapping between the identity of registered vehicle and the issued certificate.

2) Authentication Group Maintenance: In our proposed architecture, vehicle is required to register to multiple groups. Thus, it is necessary for vehicle to determine the specific group that is used for signature generation and verification. We call this group the authentication group. The authentication group is maintained by vehicle itself in a distributed way all the time.

In our proposed scheme, vehicular networks consist of nontransition zones and transition zones. In non-transition zone, vehicle receives messages only from the current group which is also the authentication group.

Transition zone is located in the border area of adjacent groups. Vehicles in transition zone may receive messages from more than one group which can come from the current group and neighboring groups. To ensure all received messages can be authenticated, the authentication in transition zone is performed by two groups, namely primary group (PG) and secondary group (SG). PG and SG together make up the authentication group of vehicle in transition zone. PG is mainly used to sign and verify message; and SG is only used to verify message that PG fails to verify.

Generally, to maximize the efficiency of authentication, the vehicle will select its current group as PG, and its neighboring group as SG. SG may contain more than one neighboring groups. Vehicle sorts these neighboring groups in an ascending order by the Euclidean distance between itself and each neigh- 


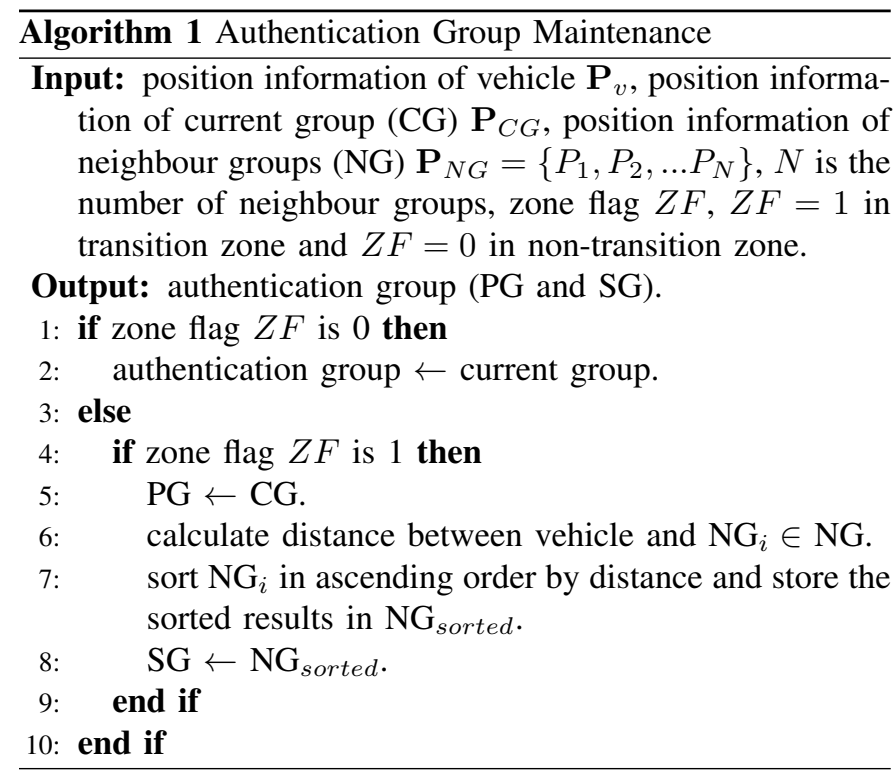

boring group. The distance is calculated based on location information. The location information of the vehicle can be obtained from GPS. The location information of the group is represented by the location of transition RSUs of the group, which can be obtained from the periodic broadcast message. The authentication group maintenance algorithm is described in Algorithm 1.

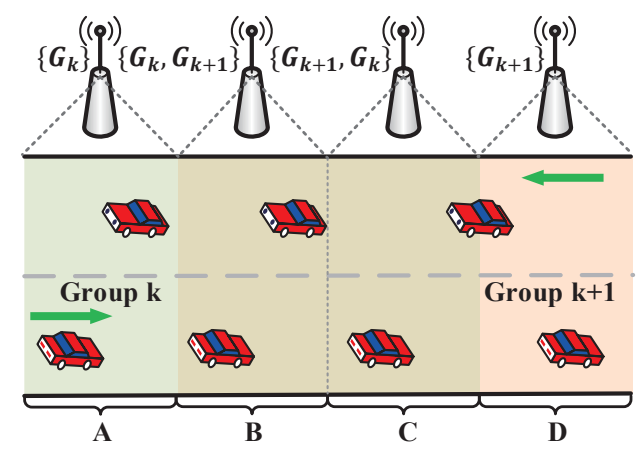

Fig. 2. Illustration of group transition.

We illustrate the group transition process using a toy example shown in Fig. 2. The example shows a vehicle driving from group $G_{k}$ into group $G_{k+1}$ and the process of completing the transition from $G_{k}$ to $G_{k+1}$. In this example, zone $\boldsymbol{A}$ and $\boldsymbol{D}$ are non-transition zone and zone $\boldsymbol{B}$ and $\boldsymbol{C}$ are transition zone. At the beginning, the vehicle is in non-transition zone $\boldsymbol{A}$. The vehicle can only receive group information from $G_{k}$ and the authentication group is $G_{k}$. As the vehicle moves into transition zone, it will receive group information from both $G_{k}$ and $G_{k+1}$. Within zone $\boldsymbol{B}$, since it is nearer to $G_{k}$ than $G_{k+1}$, it selects $G_{k}$ as its PG and $G_{k+1}$ as its SG. Once entering into zone $\boldsymbol{C}$, the distance between itself and the groups changes. It then swaps its PG and SG accordingly where its PG becomes $G_{k+1}$ and SG becomes $G_{k}$. This setting remains until the vehicle leaves zone $\boldsymbol{C}$. Upon leaving the transition zone and entering into zone $\boldsymbol{D}$, the transition process is completed and the authentication group of the vehicle is changed from $G_{k}$ to $G_{k+1}$. Table I illustrates the authentication groups of the vehicle in different zones.

TABLE I

AUTHENTICATION GROUPS IN DIFFERENT ZONES

\begin{tabular}{c|cc}
\hline \hline Zone & \multicolumn{2}{|c}{ Authentication Group } \\
\hline A & \multicolumn{2}{|c}{$G_{k}$} \\
\hline \multirow{2}{*}{ B } & PG & $G_{k}$ \\
& SG & $G_{k+1}$ \\
\hline \multirow{2}{*}{ C } & PG & $G_{k+1}$ \\
& SG & $G_{k}$ \\
\hline D & \multicolumn{3}{|c}{$G_{k+1}$} \\
\hline \hline
\end{tabular}

3) Signature Generation: In vehicular networks, vehicles interact with each other by broadcasting messages. In the message, the timestamp field is employed to record the current time. Before a vehicle sends a message, the message should be signed. The authentication group is used to generate signature. Note that in transition zone, only PG is used to sign message.

In the process of signature generation, group public key $g p k=\left(g_{1}, g_{2}, \omega\right)$, group secret key $\boldsymbol{g s} \boldsymbol{k}[i]=\left(A_{i}, x_{i}\right)$, and message $M \in\{0,1\}^{*}$ are needed. First, random values $r \stackrel{R}{\longleftarrow} \mathbb{Z}_{p}, \alpha \stackrel{R}{\longleftarrow} \mathbb{Z}_{p}$ are picked and values $u, v, T_{1}, T_{2}, \delta$ are computed as follows:

$$
\begin{aligned}
& (\tilde{u}, \tilde{v}) \leftarrow H_{0}(g p k, M, r) \in \mathcal{G}_{\mathcal{B}}{ }^{2}, \quad \delta \leftarrow x_{i} \alpha, \\
& u \leftarrow \psi(\tilde{u}), \quad v \leftarrow \psi(\tilde{v}), \quad T_{1} \leftarrow u^{\alpha}, \quad T_{2} \leftarrow A_{i} v^{\alpha},
\end{aligned}
$$

where $H_{0}$ is hash function. Then, blinding values $r_{\alpha} \stackrel{R}{\longleftarrow} \mathbb{Z}_{p}$, $r_{x} \stackrel{R}{\longleftarrow} \mathbb{Z}_{p}, r_{\delta} \stackrel{R}{\longleftarrow} \mathbb{Z}_{p}$ are picked and values $R_{1}, R_{2}, R_{3}$ are computed as follows:

$$
\begin{aligned}
& R_{1} \leftarrow u^{r_{\alpha}}, \quad R_{3} \leftarrow T_{1}^{r_{x}} \cdot u^{-r_{\delta}} \\
& R_{2} \leftarrow e\left(T_{2}, g_{2}\right)^{r_{x}} \cdot e(v, \omega)^{-r_{\alpha}} \cdot e\left(v, g_{2}\right)^{-r_{\delta}}
\end{aligned}
$$

Finally, the challenge $c$ and values $s_{\alpha}, s_{x}, s_{\delta}$ are computed as follows:

$$
\begin{aligned}
& c \leftarrow H\left(g p k, M, r, T_{1}, T_{2}, R_{1}, R_{2}, R_{3}\right) \in \mathbb{Z}_{p}, \\
& s_{\alpha} \leftarrow r_{\alpha}+c \alpha, \quad s_{x} \leftarrow r_{x}+c x_{i}, \quad s_{\delta} \leftarrow r_{\delta}+c \delta,
\end{aligned}
$$

where $H$ is hash function. With the above, the signature $\sigma \leftarrow$ $\left(r, T_{1}, T_{2}, c, s_{\alpha}, s_{x}, s_{\delta}\right)$ can be computed.

4) Verification: When a vehicle receives a message, it checks the timestamp and the validity of the message to decide whether the message can be accepted. The process of verifying the validity involves message signature $\sigma=$ $\left(r, T_{1}, T_{2}, c, s_{\alpha}, s_{x}, s_{\delta}\right)$, message $M \in\{0,1\}^{*}$, group public key $g p k=\left(g_{1}, g_{2}, \omega\right)$ and local CRL. The authentication group is used to verify message in non-transition zone. In the case of transition zone, PG is used for verification, then SG if PG fails the verification process. The message is deemed to be invalid if verification failed in both groups.

The specific verification process consists of two parts: signature check and revocation check. Signature check is used to check whether the signature $\sigma$ is valid. It first computes $\tilde{c}$ 
using the following procedures:

$$
\begin{aligned}
& (\tilde{u}, \tilde{v}) \leftarrow H_{0}(g p k, M, r) \in \mathcal{G}_{\mathcal{B}}{ }^{2}, \\
& u \leftarrow \psi(\tilde{u}), \quad v \leftarrow \psi(\tilde{v}), \\
& \tilde{R}_{1} \leftarrow u^{s_{\alpha}} / T_{1}^{c}, \quad \tilde{R}_{3} \leftarrow T_{1}^{s_{x}} u^{-s_{\delta}}, \\
& \tilde{R}_{2} \leftarrow e\left(T_{2}, g_{2}\right)^{s_{x}} e(v, \omega)^{-s_{\alpha}} e\left(v, g_{2}\right)^{-s_{\delta}} \\
& \quad \cdot\left(e\left(T_{2}, \omega\right) / e\left(g_{1}, g_{2}\right)\right)^{c}, \\
& \tilde{c} \leftarrow H\left(g p k, M, r, T_{1}, T_{2}, \tilde{R}_{1}, \tilde{R}_{2}, \tilde{R}_{3}\right) .
\end{aligned}
$$

And then checks whether $\tilde{c}$ matches $c$ which is the challenge value in $\sigma$. A valid signature should produce a matching challenge value; otherwise the signature is invalid.

Revocation check is used to check whether $\sigma$ is generated by a revoked user. The process requires computing $e\left(T_{2} / A, \tilde{u}\right)$ for each element $A$ in the local CRL. If the condition $e\left(T_{2} / A, \tilde{u}\right) \neq e\left(T_{1}, \tilde{v}\right)$ holds for all elements in the local $\mathrm{CRL}$, the signer of the message is said to be valid.

It is worth noting that the receiver accepts the message only if it passes both signature check and revocation check. Furthermore, if the message does not pass signature check, the revocation check will not proceed.

5) Revealing and Tracing: The GM is capable of revealing the signature from its group members and thus it can trace the identity of the compromised members. Only the traffic management authorities have the right to trace the identity of the malicious vehicle. To trace the identity of a vehicle, the following materials are necessary: message $M \in\{0,1\}^{*}$ and its signature $\sigma=\left(r, T_{1}, T_{2}, c, s_{\alpha}, s_{x}, s_{\delta}\right)$. In our scheme, the tracing process consists of three stages. Firstly, determining which group is used to sign the message. The traffic management authorities distribute the tracing materials to all of the GMs. Each GM verifies the signature with its public group key. If the signature passes the verification, it is indicated that the group is used to sign the message. Secondly, determining which member of the group has generated the signature. GM needs to conduct the following operations: for all $i \in[1, n]$, search for $A_{i}$ such that $e\left(T_{2} / A_{i}, \tilde{u}\right)=e\left(T_{1}, \tilde{v}\right) . A_{i}$ can be used to distinguish the group member that has generated the signature. Thirdly, obtaining the identity of the vehicle. By providing $A_{i}$ and looking up the registration table, we can obtain the identity of the vehicle.

6) CRL Management: The purpose of revocation is to prevent the malicious vehicle from further harming other legitimate vehicles. In our scheme, there are two kinds of identity that are involved in the process of revocation: the identity of the vehicle and the revocation token of the vehicle. The identity of the vehicle is unique in the whole vehicular networks, which is similar to the license plate number of the vehicle and is issued by the traffic management authority. The revocation token of a vehicle is a component of the certificate for the vehicle, which is issued to the vehicle by the group manager when the vehicle registers to the group. The revocation token of the vehicle can represent the internal identity of the vehicle in the group. In our scheme, there are multiple groups. A Vehicle owns different revocation tokens in different groups.
Based on the above two kinds of identity, we design the management mode of the two-tier CRL, which consists of the global $C R L$ and the local CRL. If a malicious vehicle needs to be revoked, the global CRL manager distributes the identity of the revoked vehicle to all group managers (GMs). Upon receiving the identity, each GM adds the identity to its global CRL. Then the GM determines whether the revoked vehicle has registered to itself. The method to determine is searching for the identity in the registration table of the group. If the revoked vehicle has registered to the group, the GM adds the revocation token of the vehicle to its local CRL. Lastly, the GM that has updated the local CRL distributes local CRL to the vehicles within its group.

The global CRL and local CRL work together to revoke malicious vehicles. The global CRL works in the GM side. When a vehicle registers to a group, the GM of the group checks whether the identity of the vehicle has been added in the global CRL. If the identity appears in global CRL, the GM will reject the registration request. The messages sent by the unregistered vehicle cannot pass the signature check of other legitimate vehicles.

The local CRL works in the vehicle side. If a vehicle has already registered in the group, the local CRL is used to revoke the vehicle. According to the local CRL of the group, each legitimate vehicle in the group checks all receiving messages. If the revocation token of a vehicle appears in the local CRL, messages sent by the vehicle will not pass the revocation check.

The behavior of revoking a vehicle is a global behavior for all groups in vehicular networks. If a vehicle is revoked, the information necessary for revocation will be distributed to all groups. The unique identity of the revoked vehicle is distributed to all group managers; the different revocation tokens of the revoked vehicle are distributed to vehicles in different groups, respectively. As a result, the vehicle will be revoked in all groups.

Formally, the relationship between the global CRL and the local CRL can be denoted as follows:

$$
C R L_{\text {local }}^{G_{k}}=M_{I D \rightarrow A_{i}^{G_{k}}}\left(C R L_{\text {global }} \cap T_{\text {reg }}^{G_{k}}\right)
$$

where $C R L_{\text {local }}$ denotes the global CRL, $C R L_{\text {local }}^{G_{k}}$ denotes the local CRL of group $G_{k}, T_{r e g}^{G_{k}}$ denotes the registration table of group $G_{k}$, and $M_{I D \rightarrow A_{i}^{G_{k}}}$ denotes the mapping function from the vehicle identity to the revocation token in group $G_{k}$.

\section{Security Analysis}

In this subsection, we shall analyze security property of our proposed authentication scheme.

1) Entity Authentication: In our scheme, there are multiple sub-areas and each sub-area is managed by one group. A vehicle may register to one or more groups based on its actual demands. The entity authentication is completed on the basis of the following rules: in the interior of a specific subarea, only the certificate issued by the group that manages the sub-area is allowed to be used by the vehicle for entity authentication; in the border of the specific sub-area, vehicle completes entity authentication by using a set of certificates, 
which are issued by the group that manage the sub-area and its neighbor groups. According to above rules, any vehicle in vehicular networks can authenticate all received messages.

2) Data Integrity: The adversary may modify the message generated by the legitimate vehicle and then broadcasts the modified message to other vehicles. The receiving vehicle of the message can detect this malicious behavior by the verification of signature. If the message, $M \in$ $\{0,1\}^{*}$, is modified, the challenge $c$ will not be equal to $H\left(\mathrm{gpk}, M, r, T_{1}, T_{2}, \tilde{R}_{1}, \tilde{R}_{2}, \tilde{R}_{3}\right)$, which results in the message failing to pass the signature check. Therefore, the integrity of the message is protected.

3) Anonymity: In our scheme, vehicles can communicate anonymously with each other. For group signature, the group public key, gpk $=\left(g_{1}, g_{2}, \omega\right)$, is employed to verify signed messages. All group members in the group share one group public key, which does not carry any information related to the identity of the vehicle. Moreover, based on Decision Linear Assumption, it is also computationally hard to obtain identity information of the sending vehicle from the signature, $\sigma=$ $\left(r, T_{1}, T_{2}, c, s_{\alpha}, s_{x}, s_{\delta}\right)$. Therefore, the identity privacy of the vehicle can be preserved.

4) Unlinkability: Under Decision Linear Assumption, the process of verification is zero-knowledge [21]. The receiving vehicle cannot reveal any useful information about the sender from the signature. Therefore, the receiving vehicle is unable to relate two different signatures even if they are generated from the same sender.

5) Traceability: Compared with the underlying group signature scheme [13], we introduce an extra stage for our scheme to implement the traceability. The materials necessary for tracing consist of a message and the signature for the message. The extra stage is to determine which group has been used by the traced vehicle to sign the message. The task of determining the group is undertaken commonly by all group managers. Each group manager verifies in parallel the signature of the message with its group public key. If the signature passes the verification by a group, this group will be the group that has been used by the traced vehicle. Then, the remaining two stages for tracing will be also completed by this group. The stage of determining the group is the same as signature verification process which is secure.

6) Revocation: In our scheme, we aim to reduce the revocation check delay by decreasing the number of revoked vehicles that are checked in the process of revocation check. From the perspective of single group, we can divide the revoked vehicles into two parts: a) the revoked vehicles that have registered to the group, and b) the revoked vehicles that have not registered to the group. In the process of revocation check, vehicles only need to check the revoked vehicles that have registered, so the revocation check delay can be reduced. Yet, we do not neglect the revoked vehicles that have not registered. We mitigate the task of checking this part of revoked vehicles from the side of vehicles to the side of group manager. Therefore, the method in our scheme reduces the revocation check delay and does not affect the security in the aspect of revocation.

7) Resistance to On-off Attack: In an on-off attack, if a malicious vehicle behaves badly (the state of on), it will be revoked from the vehicular communication system by adding it to the CRL. For the malicious vehicle, the revocation will cause the following consequences: the group manager refuses to issue new certificate to the malicious vehicle, and other normal vehicles refuse to trust the messages sent by the malicious vehicle. As a result, the malicious vehicle cannot continue to deceive the vehicular communication system by performing good behaviors (the state of off) to obtain trust.

8) Resistance to Message Spoofing Attack: A malicious vehicle may broadcast fake messages which may disturb the traffic order. In our scheme, the legitimate vehicles validate the received messages by signature checking, and only the messages that pass the checking can be accepted. To generate a valid signature for a message, there are three essential elements: the message, the group public key, and the group secret key. The group public key and secret key are issued confidentially to legitimate vehicles by the group manager when the vehicle registers to the group. Due to lack of the group public key and the secret key, the malicious vehicle cannot generate valid signature and cannot pass the checking. Finally, the messages broadcasted by malicious vehicle will be deemed invalid and be discarded.

9) Resistance to Replay Attack: The malicious vehicle may store messages broadcasted by legitimate vehicles in the past, and replay them to cause the receivers to react to non-existing road condition. In our proposed scheme, the timestamp field is included in the message to keep the freshness. When sending a message, the sender adds the current time into the timestamp field and then signs the message. Once received the message, the receiving vehicle will firstly validate the signature to ensure the integrity of the message. Then, the receiving vehicle will check the timestamp of the message and decide whether to take actions based on the freshness of the message. By this way, the message including the road condition information in the past can be detected and the replay attack can be resisted.

\section{Area Division in FEDAS}

In our proposed authentication scheme, the entire area is divided into multiple sub-areas. Each sub-area is managed by one group. For delay consideration, ideally the size of the sub-area should be set as small as possible. However, if the size is set too small, management overhead of sub-areas increases due to excessive sub-areas and transition zones. To determine an adequate size for sub-areas, we shall formulate it as an optimization problem with an objective to minimize the number of groups to avoid excessive overhead yet to maintain delay within a target.

\section{A. Problem Formulation}

The road topology in vehicular networks can be modeled as graph theory [41]. We use a directed graph $\hat{G}=(V, E)$ to express the road topology, where $V=\left\{v \mid v=v_{p}, p=\right.$ $1,2, \ldots, P\}$ is the set of intersections (i.e. vertexes), $E=$ $\left\{e \mid e=e_{j}, j=1,2, \ldots, J\right\}$ is the set of roads (i.e. edges), $P$ and $J$ denote the total number of vertexes and edges, respectively. The task of area division is to divide the entire area into multiple sub-areas, i.e., partition the road topology 
TABLE II

NOTATIONS USED IN THE AREA DIVISION.

\begin{tabular}{|c|c|}
\hline Symbol & Description \\
\hline$L$ & The local CRL length threshold \\
\hline$G_{k}$ & The group that manages the $k$-th sub-area \\
\hline$\hat{G_{k}}$ & $\begin{array}{l}\text { The sub-graph whose edges are managed by group } \\
G_{k}\end{array}$ \\
\hline$N$ & $\begin{array}{l}\text { The average number of the group members that have } \\
\text { registered }\end{array}$ \\
\hline$N^{\prime}$ & The average number of vehicles in a given sub-area \\
\hline$T$ & The validity period of the certificate \\
\hline$T^{\prime}$ & The mean sojourn time for vehicle in the system \\
\hline$Q\left(\hat{G_{k}}\right)$ & $\begin{array}{l}\text { The total traffic volume that comes from the exterior } \\
\text { of a given sub-area }\end{array}$ \\
\hline$\eta$ & $\begin{array}{l}\text { The ratio of the traffic volume that do not have a } \\
\text { valid certificate to the total traffic volume }\end{array}$ \\
\hline$\lambda$ & The average total registration rate \\
\hline$\lambda_{1}$ & $\begin{array}{l}\text { The average registration rate in the border of a given } \\
\text { sub-area }\end{array}$ \\
\hline$\lambda_{2}$ & $\begin{array}{l}\text { The average registration rate in the interior of a given } \\
\text { sub-area }\end{array}$ \\
\hline$l(e)$ & The length of the edge $e$ \\
\hline$d(e)$ & The density of vehicles on the edge $e$ \\
\hline$\alpha$ & $\begin{array}{l}\text { The ratio of the number of revoked members to the } \\
\text { number of total members in the group }\end{array}$ \\
\hline$f$ & $\begin{array}{l}\text { The average frequency of vehicle entering a given } \\
\text { sub-area in single validity period of the certificate }\end{array}$ \\
\hline
\end{tabular}

graph into multiple connected sub-graphs. Let $\mathbb{N}$ be an instance of a partition of graph $\hat{G}$ and $|\mathbb{N}|$ be the number of connected sub-graphs in the partition. Let $\mathbb{M}$ be a set containing all such instances. For a particular instance, say $\mathbb{N}$, of the partition, we denote $\hat{G}_{k}, k=1,2, \ldots,|\mathbb{N}|$, to be the $k$-th connected subgraph, where $|\mathbb{N}|$ is the total number of sub-graphs in the partition. For each sub-area, there is a group established to be responsible for providing authentication services. We denote $G_{k}, k=1,2, \ldots,|\mathbb{N}|$, to be the group managing the sub-area $\hat{G}_{k}$. If edge $e_{j}$ is allocated to sub-area $\hat{G}_{k}$, it will be managed by group $G_{k}$.

Our area division problem is a graph partition problem with an objective to obtain the least number of connected sub-graphs where each sub-graph has no more than a certain local CRL length threshold, $L$. The optimization problem is described as follow.

$$
\begin{aligned}
\min _{\mathbb{N} \in \mathbb{M}} & |\mathbb{N}| \\
\text { s.t. } & \max _{k} h\left(G_{k}\right) \leq L, k=1,2, \ldots,|\mathbb{N}|
\end{aligned}
$$

where function $h\left(G_{k}\right)=\overline{\left|C R L_{\text {local }}^{G_{k}}\right|}$ denotes the average length of local CRL in group $G_{k}$.

\section{B. Modeling of Average local CRL Length}

To find the solution for the area division problem, it is critical to determine the length of local CRL. We shall establish the relationship between length of local CRL and information of traffic volume for a given sub-area. Without loss of generality, we consider a particular sub-area, say $\hat{G}_{k}$ managed by the GM of group $G_{k}$. We note that the average length of local CRL in $G_{k}$ is proportion to the number of group members in $G_{k}$. We shall obtain the average length of local CRL by first calculating the number of group members of $G_{k}$.

A vehicle becomes a member of $G_{k}$ by registering itself to the GM of $G_{k}$ to obtain a certificate. The registration occurs when a vehicle enters $\hat{G}_{k}$ without a valid certificate, or its certificate expires while traveling within $\hat{G}_{k}$. In other words, the registration may occur (i) at the border of $\hat{G_{k}}$, or (ii) within the interior of $\hat{G}_{k}$. Fig. 3 illustrates the vehicle registration model in a particular sub-area, say $\hat{G}_{k}$. In the figure, the vehicle arrives at the border of the $\hat{G_{k}}$ first, then drives into the interior of $\hat{G}_{k}$, and goes across $\hat{G}_{k}$ before leaving $\hat{G}_{k}$.

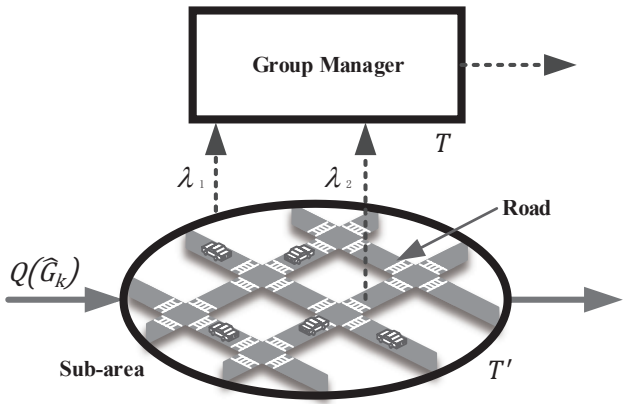

Fig. 3. The vehicle registration model.

We use a queuing system to describe the evolution of the number of certificates in group $G_{k}$. Let $N$ be the average number of certificates in $G_{k}$. Note that it is also the number of registered group members in $G_{k}$ since each registered vehicle carries exactly one certificate. The creation of certificates depends on the arrival of vehicle registration request. Let $\lambda$ be the arrival rate of the registration requests and $T$ be the validity period of a certificate. By Little's Theorem [42], we have

$$
N=\lambda T \text {. }
$$

We further use $\lambda_{1}, \lambda_{2}$ to denote the registration arrival rate at the border of $\hat{G}_{k}$ and within the interior of $\hat{G}_{k}$, respectively. We have

$$
\lambda=\lambda_{1}+\lambda_{2} .
$$

To compute $\lambda_{1}$ and $\lambda_{2}$, we define $Q\left(\hat{G}_{k}\right)$ to be the total traffic arriving from the exterior into $\hat{G}_{k}$, and $\eta$ to be the fraction of traffic without a valid certificate. We immediately get

$$
\lambda_{1}=\eta Q\left(\hat{G_{k}}\right) .
$$

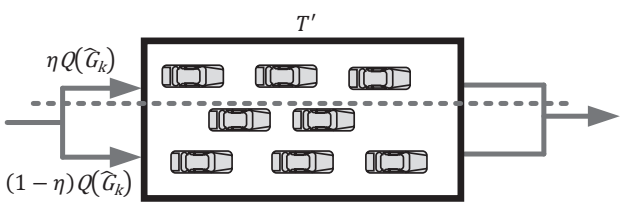

Fig. 4. The constitution for vehicles in $\hat{G}_{k}$. 
We now build another queuing system describing the number of vehicles in $\hat{G}_{k}$, as shown in Fig. 4. Let $N^{\prime}$ be the average number of vehicles in $\hat{G}_{k}$ and $T^{\prime}$ be the mean sojourn time of a vehicle in $\hat{G}_{k}$. Then by Little's Theorem, the average number of vehicles in $\hat{G}_{k}$ is

$$
\begin{aligned}
N^{\prime} & =Q\left(\hat{G}_{k}\right) T^{\prime} \\
& =\eta Q\left(\hat{G}_{k}\right) T^{\prime}+(1-\eta) Q\left(\hat{G}_{k}\right) T^{\prime} .
\end{aligned}
$$

In the above, the first term corresponds to the vehicles obtaining a new certificate when entering $\hat{G}_{k}$ and the second term corresponds to those already carrying a valid a certificate. For those obtaining a new certificate, the remaining lifetime of the certificate is simply $T$. Since the certificate lifetime is usually much longer than the sojourn time of those vehicles in $\hat{G}_{k}$, they do not trigger another registration request within the interior.

For those vehicles already carrying a valid certificate, some of these certificates may expire which then triggers registration requests within the interior of $\hat{G}_{k}$. We assume that the residual lifetime of these certificates is uniformly distributed in the time interval $(0, T)$. If the sojourn time of those vehicles is longer than their certificate residual lifetime, they trigger a registration request. Because these vehicles are independent, the number of vehicles triggering a registration request per unit time follows Binomial distribution with parameters $\left((1-\eta) Q\left(\hat{G}_{k}\right) T^{\prime}, \frac{1}{T}\right)$, the registration arrival rate is thus

$$
\lambda_{2}=\frac{(1-\eta) Q\left(\hat{G}_{k}\right) T^{\prime}}{T} .
$$

Substituting (4), (5), (6) and (7) into (3), we can get

$$
\begin{aligned}
N & =\eta Q\left(\hat{G}_{k}\right) T+(1-\eta) Q\left(\hat{G}_{k}\right) T^{\prime} \\
& =\eta Q\left(\hat{G}_{k}\right) T+(1-\eta) N^{\prime} .
\end{aligned}
$$

We further know that the average number of vehicles in $\hat{G}_{k}$, or $N^{\prime}$ also depends on the length of the roads and density of vehicles. Let $l(e)$ denote the length of edge $e$, and $d(e)$ denote the density of vehicles on the edge $e$, where $e \in \hat{G}_{k}$, then

$$
N^{\prime}=\sum_{e \in \hat{G}_{k}} l(e) d(e)
$$

Finally, we can obtain the average length of local CRL for group $G_{k}$ by

$$
\begin{aligned}
h\left(G_{k}\right) & =\alpha N \\
& =\alpha\left(\eta Q\left(\hat{G}_{k}\right) T+(1-\eta) \sum_{e \in G_{k}} l(e) d(e)\right)
\end{aligned}
$$

where $\alpha$ is the ratio of the number of revoked members to the number of total members in the group.

\section{Modeling of Traffic Volume}

We shall now focus on determining the total traffic volume in $\hat{G}_{k}$. Vehicles enter into $\hat{G}_{k}$ via the intersections. Vehicles may enter from either another road connected to the intersection, or directly from other non-road zones, such as parking lots of residential district, the business district, or schools.
Similarly, when vehicles leave the intersection, they can be also enter into another road, or non-road zones.

Sub-area $\hat{G}_{k}$ is managed by group $G_{k}$, and it can be represented by a graph $\left(V_{G_{k}}, E_{G_{k}}\right)$, where $V_{G_{k}}$ is the set of intersections in the selected sub-area and $E_{G_{k}}$ is the set of roads in $\hat{G}_{k}$. Fig. 5 shows the arrival and departure traffic at an intersection. Roads which are represented by edges can be categorized into ingress edges and egress edges. The ingress edges are the roads on which vehicles move into the intersection, and egress edges are those where vehicles leave the intersection. In Fig. 5, $e_{v}^{i n}(k), k=1,2, \ldots, m$, is used to denote the $k$-th ingress edge of the intersection $v \in V_{G_{k}}$, and $e_{v}^{\text {out }}(k), k=1,2, \ldots, n$, the $k$-th egress edge. Here, $m$ is the total number of ingress edges for $v$, and $n$ is the total number of egress edges for $v$. For the intersection $v \in V_{G_{k}}$, let us denote the ingress edge set by $S_{v}^{i n}=\left\{e \mid e=e_{v}^{i n}(k), k=1,2, \ldots, m\right\}$, and the egress edge set by $S_{v}^{\text {out }}=\left\{e \mid e=e_{v}^{\text {out }}(k), k=1,2, \ldots, n\right\}$.

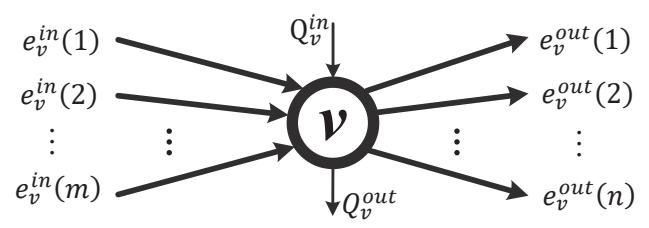

Fig. 5. The traffic volume model in the intersection.

We use $q(e)$ to denote the traffic volume on edge $e \in E$. For example, $q\left(e_{v}^{i n}(\cdot)\right)$ denotes the traffic volume for the edge $e_{v}^{i n}(\cdot)$. We use $Q_{v}^{i n}$ to denote the traffic volume that comes directly from non-road zones in intersection $v$, and $Q_{v}^{\text {out }}$ denotes the traffic volume that enters non-road zones from intersection $v$.

Let $Q\left(v \rightarrow G_{k}\right)$ denote the ingress traffic volume that enters $\hat{G_{k}}$ via intersection $v \in V_{G_{k}} . Q\left(v \rightarrow G_{k}\right)$ can be determined by:

$$
Q\left(v \rightarrow G_{k}\right)=\left\{\begin{array}{cl}
\sum_{e_{v}^{i n} \in E_{v}^{\prime}} q\left(e_{v}^{i n}\right)+Q_{v}^{i n}, & v \in V_{G_{k}}^{\text {border }} \\
Q_{v}^{i n} & , v \in V_{G_{k}}-V_{G_{k}}^{\text {border }}
\end{array}\right.
$$

where $E_{v}^{\prime}=\left\{e \mid e \in S_{v}^{i n}\right.$ and $\left.e \notin E_{G_{k}}\right\}, V_{G_{k}}^{\text {border }}$ is the set of the intersection situated at the border of $\hat{G}_{k}$.

The total traffic volume in $\hat{G}_{k}$, or $Q\left(\hat{G}_{k}\right)$, is the sum of the ingress traffic volume of all intersections in $\hat{G}_{k}$. That is

$$
Q\left(\hat{G}_{k}\right)=\sum_{v \in V_{G_{k}}} Q\left(v \rightarrow G_{k}\right)
$$

\section{Determination of $\eta$}

In this section, we shall determine $\eta$. Recall that $\eta$ is the ratio of the traffic volume without a valid certificate to the total traffic volume. Vehicles that carry a valid certificate must have visited the same sub-area earlier. We denote $f$ to be the revisiting rate, which is defined as the average frequency of a vehicle revisiting the same sub-area during the lifetime of a certificate. We shall determine $\eta$ via $f$. 
Focusing on the interior of a particular sub-area $\hat{G}_{k}$, we know that $T^{\prime}$ is the average sojourn time in $\hat{G_{k}}$, and $f$ is the average number of vehicle entering $\hat{G}_{k}$ during the lifetime of a certificate which is $T$. Therefore the probability, denote $P_{i n}$, that a vehicle is found in $\hat{G}_{k}$ is

$$
P_{\text {in }}=\frac{f T^{\prime}}{T}
$$

and the probability, denote $P_{\text {out }}$, that a vehicle is found outside of $\hat{G_{k}}$ is

$$
P_{\text {out }}=1-\frac{f T^{\prime}}{T}
$$

Given the total number of vehicles $N$, we can further determine the average numbers of vehicles inside of and outside of $\hat{G}_{k}$ by

$$
N \cdot P_{i n}=\frac{N f T^{\prime}}{T}
$$

and

$$
N \cdot P_{\text {out }}=N\left(1-\frac{f T^{\prime}}{T}\right)
$$

respectively.

Moreover, by (6) and (8), the average numbers of vehicles inside of and outside of $\hat{G}_{k}$ are $N^{\prime}=Q\left(\hat{G}_{k}\right) T^{\prime}$, and $N-N^{\prime}=$ $\eta Q\left(\hat{G}_{k}\right)\left(T-T^{\prime}\right)$, respectively. Combining the above results, we have

$$
\frac{N f T^{\prime} / T}{N\left(1-f T^{\prime} / T\right)}=\frac{N^{\prime}}{N-N^{\prime}}=\frac{Q\left(\hat{G}_{k}\right) T^{\prime}}{\eta Q\left(\hat{G}_{k}\right)\left(T-T^{\prime}\right)}
$$

which leads to

$$
\eta=\frac{T-f T^{\prime}}{f\left(T-T^{\prime}\right)} .
$$

From the final result, we see that determination of $\eta$ requires $f$. While there is no existing model describing $f$, fortunately some government documents share related information in the public domain. One example is the Annual Report for Transport of Beijing (2019) [43] where statistical information describing traffic activities of different types of vehicles is given. We can then consolidate the provided information for different types of vehicles to compute $f$ by

$$
f=\sum_{k=1}^{K} \xi_{k} f_{k}
$$

where $k$ is the type of vehicles such as cars, buses, taxis, trucks, $K$ is the total number of different vehicle types, $k=$ $1,2, \ldots, K, f_{k}$ denotes the average frequency of the vehicles of type $k$ entering a particular sub-area, $\xi_{k}$ denotes the proportion of the type $k$ vehicles in the total traffic volume.

\section{E. Area Division Algorithm}

In this section, we propose a heuristic method to find a suboptimal solution of the problem formulated in Section V-A. The heuristic method, depicted in Algorithm 2, proceeds as follows. The design of the algorithm is similar to a full-bin packing algorithm in bin packing problem where a connected

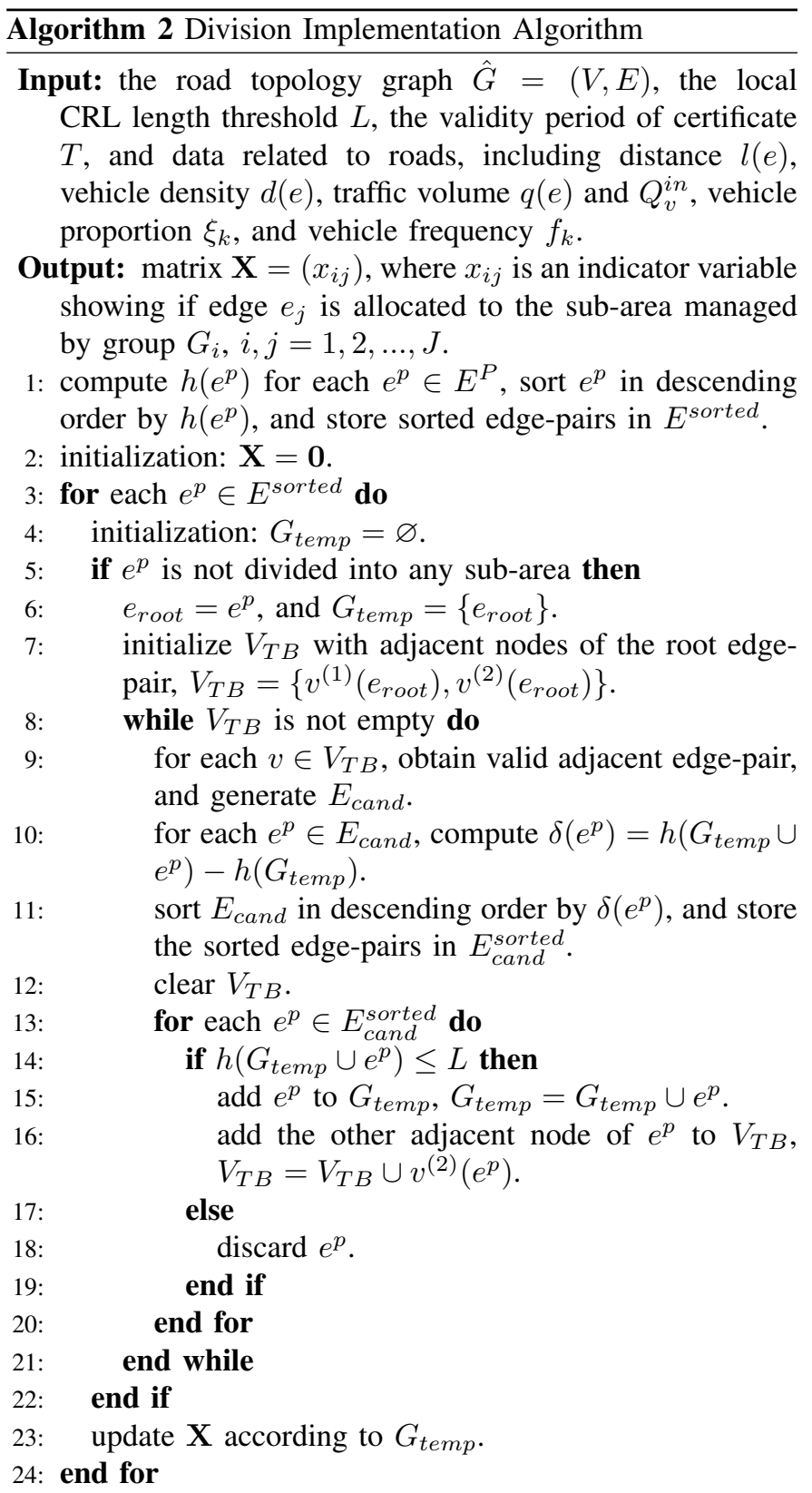

sub-graph is a bin, the local CRL length threshold is the bin size, and edges are items to pack into a bin.

The algorithm takes the road topology graph and other related parameters as input, and the output is the matrix that reflects the relationship between sub-areas and edges. The process of the division considers the road as a dividing unit, which is also called edge-pair, $e^{p}$. For a two-way road, edgepair consists of two edges; and for one-way road, edge-pair consists of one edge. All edge-pairs in the topology form the edge-pair set, $E^{P}$. The establishment of each sub-area starts from the root edge-pair, $e_{\text {root }}$, then expands by Breadth First Search (BFS), and ends until the local CRL length threshold is satisfied. The root edge-pair is selected from the sorted edgepair sequence, which is sorted by the length of local CRL for the edge-pair, $h\left(e^{p}\right)$. The process of BFS involves two sets, i.e. the set of nodes, $V_{T B}$, and the set of edge-pairs, $E_{c a n d}$. 
The set $V_{T B}$ stores the nodes in a temporary boundary of the sub-area during the establishment of the sub-area, which is used to generate $E_{\text {cand }}$. $E_{\text {cand }}$ is the candidate edge-pair set that prepares for the edge addition. To qualify for a candidate, the edge-pair is required to satisfy three conditions, where the edge-pair has (i) not been divided, (ii) not been discarded, and (iii) not been selected as a candidate before. Through the establishment of a sub-area, all the divided edges are stored temporarily in $G_{t e m p}$. When the establishment of the sub-area completes, $X$ is updated according to $G_{t e m p}$. The algorithm ends when all edges are allocated.

\section{Vi. Performance Evaluation}

In this section, we evaluate the performance of the privacypreserving delay-aware authentication scheme. Our scheme can decrease the delay and the decreased delay is contributed by the use of shorter local CRL. To validate this, we first divide the road topology in real world by using the method of area division proposed in Section V. Then, we study the impact of area division on the length of CRL. Finally, we test the delay performance in the simulation environment.

\section{A. Simulation Settings}

The simulation is performed in the scenario with the road topology illustrated in Fig. 6. The size of the topology is $5.36 \mathrm{~km}$ by $2.13 \mathrm{~km}$ and the total length of the road in the topology is $104.38 \mathrm{~km}$. The mobility models of vehicles in the simulation are generated by SUMO [44]. The source and destination of the trip for vehicle are selected randomly, and the route between source and destination is generated based on the shortest path principle. The maximum vehicle speed is limited by the speed limit of the road, i.e. $60 \mathrm{~km} / \mathrm{h}$. The statistical data related to the road that are necessary for area division are obtained from SUMO. In the simulation, we set up three types with different values of the total numbers of vehicles, namely 3000, 6000 and 9000 . The validity period of the certificate is one month. The delay performance is evaluated by NS3 [45]. Vehicles and RSUs use 802.11p as their MAC protocol and the wireless channel capacity is $6 \mathrm{Mbps}$. Vehicles broadcast messages every 300 milliseconds. RSUs broadcast periodically group information and the interval time is 2 seconds. Each simulation session lasts for 100 seconds. The parameters above are summarized in Table III.

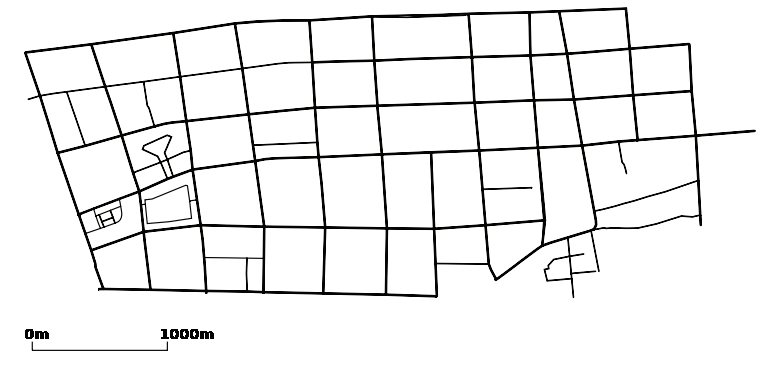

Fig. 6. The road topology.

For parameters $\xi_{k}$ and $f_{k}, k=1,2, \ldots, K$, we set the value based on the Annual Report for Transport of Beijing (2019)
TABLE III

SIMULATION PARAMETERS

\begin{tabular}{l|l}
\hline \hline Parameters & Value \\
\hline Simulation area & $5.36 \mathrm{~km} \times 2.13 \mathrm{~km}$ \\
Maximum vehicle speed & $60 \mathrm{~km} / \mathrm{h}$ \\
Total number of vehicles & $3000,6000,9000$ vehicles \\
MAC protocol & $802.11 \mathrm{p}$ \\
Channel capacity & $6 \mathrm{Mb} / \mathrm{s}$ \\
Communication range & $300 \mathrm{~m}$ \\
Vehicle broadcast interval & $300 \mathrm{msec}$ \\
RSU broadcast interval & $2 \mathrm{sec}$ \\
Simulation time & $100 \mathrm{sec}$ \\
\hline \hline
\end{tabular}

[43]. The value of parameters is given in Table IV, where $f_{k}^{\prime}$ denotes the average frequency of the $k$-th type of vehicle entering the selected area in one day, $f_{k}=f_{k}^{\prime} \cdot T$.

TABLE IV

PARAMETER $\xi_{k}$ AND $f_{k}^{\prime}$

\begin{tabular}{c|ccccc}
\hline \hline Vehicle Type & car & taxi & bus & truck & others \\
\hline$\xi_{\mathbf{k}}$ & 0.8 & 0.06 & 0.02 & 0.07 & 0.05 \\
\hline $\mathbf{f}_{\mathbf{k}}^{\prime}$ & 2.8 & 5 & 4 & 2 & 0.1 \\
\hline \hline
\end{tabular}

We measure the timing of exponentiation operations and pairing operations, which will be later used to evaluate the performance of the proposed scheme. The Pairing-Based Cryptography (PBC) library [46] is used to perform the measurements. This library is a free $\mathrm{C}$ library which is designed to implement the pairing-based cryptosystems. In our measurements, we select the pairing-friendly MNT curves [47] which have been implemented in the PBC library. The hardware platform that performs the measurements is a Lenovo Thinkcentre desktop, equipped with an Intel(R) Core(TM) i5-4590 CPU @3.30GHz processor. The libraries for this platform are compiled with the GCC [48] with its version 5.4.0 in Ubuntu 16.04. The measurement results are given in Table $\mathrm{V}$, which are the average value of 1000 times for every operation, respectively.

TABLE V

TIMINGS OF DIFFERENT OPERATIONS

\begin{tabular}{c|c|c|c}
\hline \hline Operations & $\begin{array}{c}\text { Exponentiation } \\
\text { in } \mathcal{G}_{\mathcal{A}}\end{array}$ & $\begin{array}{c}\text { Exponentiation } \\
\text { in } \mathcal{G}_{\mathcal{T}}\end{array}$ & Pairing \\
\hline Time (ms) & $T_{E}^{\mathcal{G}_{\mathcal{A}}}=0.507$ & $T_{E}^{\mathcal{G}}=0.872$ & $T_{P}=2.246$ \\
\hline \hline
\end{tabular}

\section{B. Impact on the Number of Groups}

We divide the area shown in Fig. 6 into sub-areas by using Division Implementation Algorithm (that is, Algorithm 2) and related data that are necessary for area division. Fig. 7 shows the division results in three cases with three different values of the total number of vehicles. In Fig. 7, the results are shown by using the double logarithmic coordinates, the horizontal axis is the local CRL length threshold, and the vertical axis is the number of groups (or sub-areas) in the area. We can see from the figure that, as the local CRL length threshold increases, 
the number of groups decreases. Moreover, we can also see from Fig. 7 that the total number of vehicles can have direct impact to division results. With a fixed threshold, increasing the total number of vehicles leads to an increase in the number of groups.

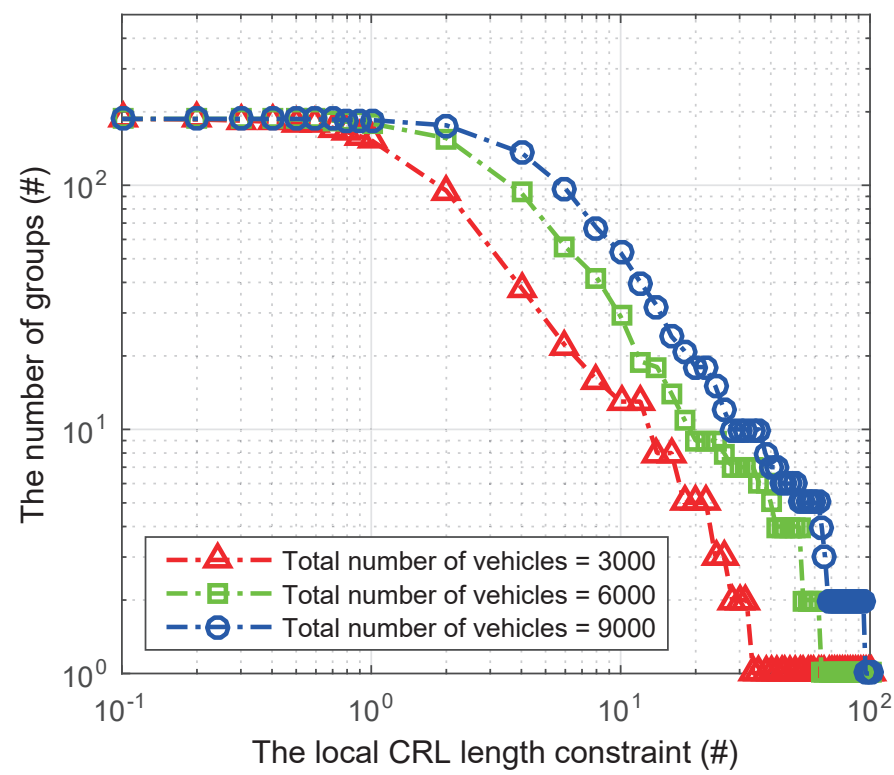

Fig. 7. The number of groups versus the local CRL length constraint. When the local CRL length for a sub-area falls below one, it indicates that the sub-area has no revoked vehicle during some period of time.

\section{The Length of $C R L$}

In this section, we shall study the impact of area division on the length of CRL. In the simulation, We set the number of revoked vehicles in the whole area to be 30,60 , and 90 for corresponding total number of vehicles, 3000, 6000, 9000, respectively. The revoked vehicles are randomly picked from all vehicles. In our design, the length of CRL is the number of revoked vehicles. The length of global CRL is the number of revoked vehicles for the whole area, and the length of local CRL is the number of revoked vehicles for the sub-area.

Here, we use the number of groups to represent the different types of division results. For each division result, there are more than one groups in the whole area. Therefore, the length of local CRL is the average value of the local CRL length for all groups in the whole area, i.e.,

$$
\overline{\left|C R L_{\text {local }}\right|}=\frac{1}{W} \sum_{k=1}^{W}\left|C R L_{\text {local }}^{G_{k}}\right|
$$

where $W$ is the number of groups and $\left|C R L_{\text {local }}^{G_{k}}\right|$ is the length of local CRL for group $G_{k}, k=1,2, \ldots W$.

Fig. 8 shows the length of CRL versus the number of groups for different total numbers of vehicles. In the figure, as the number of groups increases, the length of local CRL decreases and the length of global CRL remains constant. We also observe a sharp drop in the length of local CRL as we begin to divide an area into multiple groups. This clearly shows the performance advantage of using multiple groups.

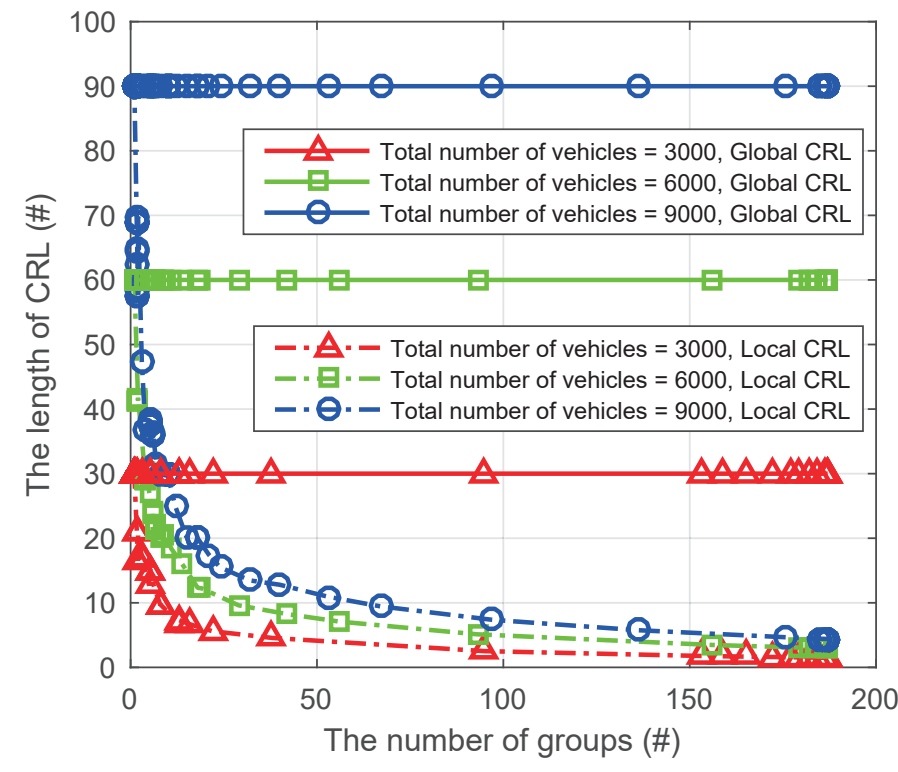

Fig. 8. The length of CRL versus the number of groups.

However, excessively dividing an area into many groups does not always bring proportionate benefits since the length of local CRL turns flat when the number of groups becomes high.

\section{End-to-End Delay Performance}

We now focus on the end-to-end delay performance of our scheme. In Section VI-C, we have obtained the global CRL for the whole area and the local CRL for each sub-area of the division results, which are necessary for the end-to-end delay simulation experiments. We also input these results into the NS3 simulation platform to test the delay performance.

The end-to-end delay is defined as the time taken for a message to be transmitted from a sender to a receiver. The end-to-end delay, $D$, consists of three parts:

$$
D=D_{t}+D_{s}+D_{v},
$$

where $D_{t}, D_{s}$ and $D_{v}$ are the transmission delay, signing delay and signature verification delay, respectively. Moreover, the process of signature verification includes two stages, which are signature check and revocation check. In other words, $D_{v}=$ $D_{s c}+D_{r c}$ where $D_{s c}$ and $D_{r c}$ are the signature check delay and revocation check delay respectively.

In the end-to-end delay, apart from transmission delay which depends on the network, all others are related to cryptographic processing. Among the cryptographic operations of group signature, the most time-consuming operations are the pairing and exponentiation [11]. In FEDAS, the delay generated by these two types of operations in the process of signing, signature check, and revocation check is given as follows:

$$
\begin{aligned}
& D_{s}=5 \cdot T_{E}^{\mathcal{G}_{\mathcal{A}}}+3 \cdot T_{E}^{\mathcal{G}_{\mathcal{T}}}+3 \cdot T_{P}, \\
& D_{s c}=\theta \cdot\left(4 \cdot T_{E}^{\mathcal{G}_{\mathcal{A}}}+4 \cdot T_{E}^{\mathcal{G}}+5 \cdot T_{P}\right), \\
& D_{r c}=\left(1+\left|C R L_{\text {local }}^{G_{k}}\right|\right) \cdot T_{P},
\end{aligned}
$$

where $T_{E}^{\mathcal{G}_{\mathcal{A}}}$ denotes the time taken by one exponentiation operation in $\mathcal{G}_{\mathcal{A}}, T_{E}^{\mathcal{G}} \mathcal{T}$ denotes the time taken by one exponentiation 


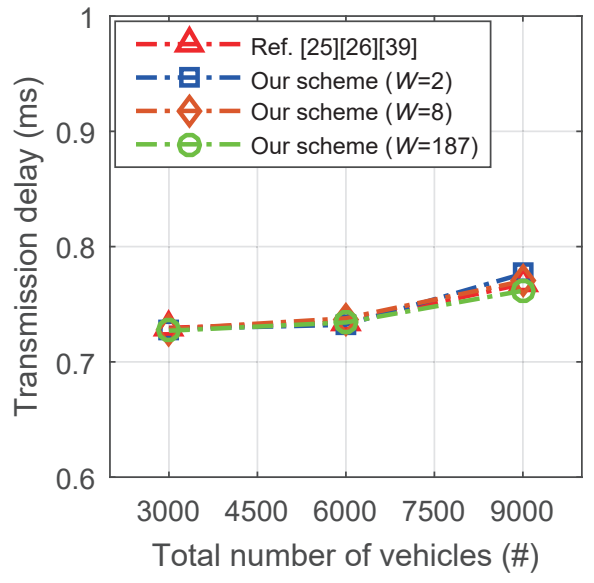

(a) Transmission delay

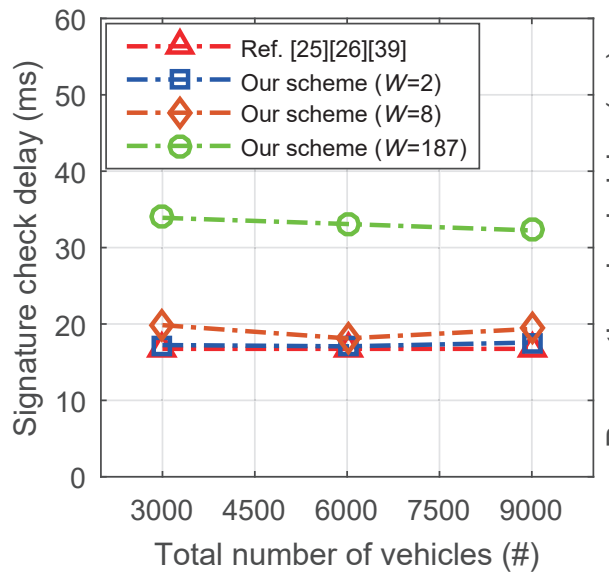

(b) Signature check delay

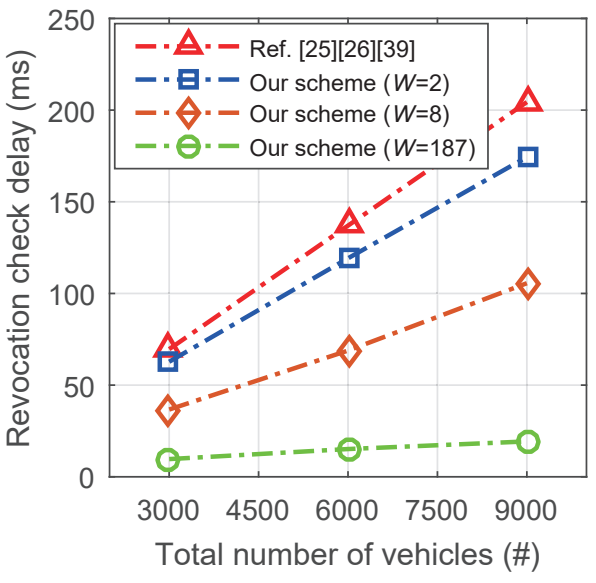

(c) Revocation check delay

Fig. 9. Individual time duration for the process contributing to the end-to-end delay. In the figure, $W$ denotes the number of groups. $W=2$ is the minimum number of groups in our scheme, and $W=187$ is the maximum number of groups that is allowed in our scheme for the road topology used in the simulation.

operation in $\mathcal{G}_{\mathcal{T}}$, and $T_{P}$ denotes the time taken by one pairing operation. The quantity $\left|C R L_{\text {local }}^{G_{k}}\right|$ is the length of local CRL for $G_{k}$. The quantity, $\theta \in \mathbb{N}^{+}$, denotes the number of signature check. We have $\theta=1$ if vehicle is located in a non-transition zone. The situation of $\theta>1$ may happen in transition zone.

In Fig. 9, we compare our scheme with the scheme in [25], [26], [39] in terms of the individual time duration for each process contributing to the end-to-end delay, except for signing delay which is a fixed value of $11.889 \mathrm{~ms}$ based on the simulation. It can be seen from Fig. 9a that the transmission delay remains the same for different schemes, it is only affected by the number of vehicles. Higher vehicle density creates more contention on the channel which leads to increased transmission delay. As shown in Fig. 9b, our scheme has higher signature check delay than scheme in [25], [26], [39]. This is because we introduce more groups in our scheme. More groups lead to more transition zones in the network, and signature check delay in transition zone is higher than that in non-transition zone. As can be seen in Fig. 9c, our scheme has lower revocation check delay than those schemes in [25], [26], [39]. The reason is that we use the shorter local CRL for our scheme and yet others use global CRL.

In Fig. 10, we compare the end-to-end delay performance of our scheme with the schemes in [25], [26], [39]. We can see that our scheme has lower end-to-end delay than those in [25], [26], [39]. When the total number of vehicles is 6000 , our scheme reduces the end-to-end delay by at least $17.5 \mathrm{~ms}(W=2)$ and at most $105.5 \mathrm{~ms}(W=187)$. Although our scheme introduces extra signature check delay, it reduces more revocation check delay. As a result, the overall end-toend delay is lower. Focusing on the signature check delay and revocation check delay of our scheme $(W=187)$ and comparing with other schemes when the total number of vehicles is 6000, we see from Fig. 9 that while our scheme increases the signature check delay by $16.3 \mathrm{~ms}$, the revocation check delay is decreased by $121.8 \mathrm{~ms}$. As a result, the overall end-to-end delay is reduced by $105.5 \mathrm{~ms}$.

Furthermore, authors in [39] propose to setup a threshold

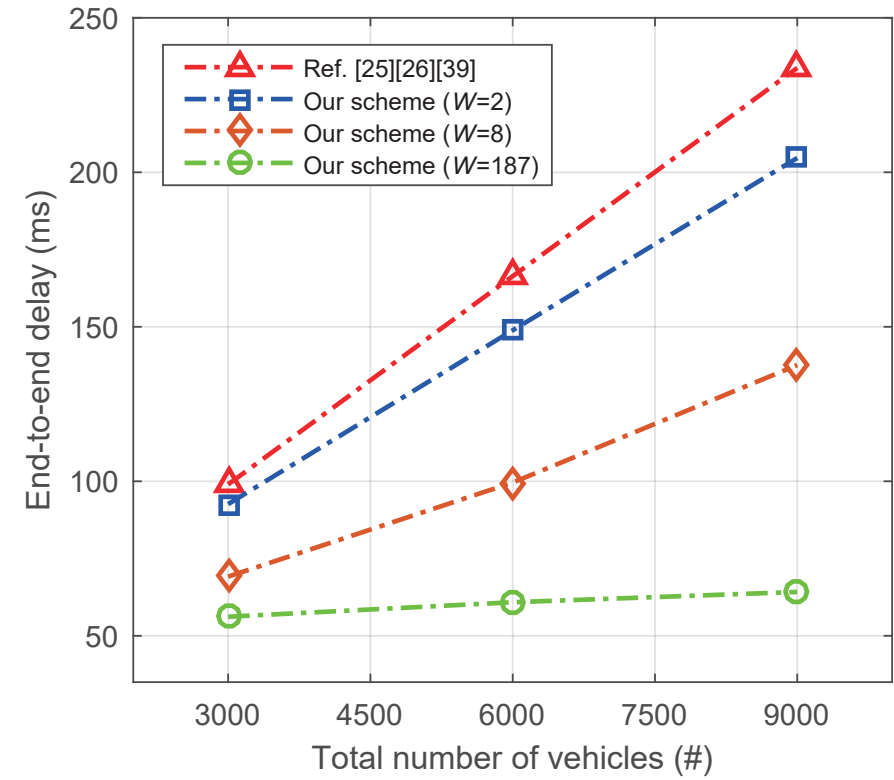

Fig. 10. End-to-end delay. In the figure, $W$ denotes the same meaning with that in Fig. 9.

for the method of revocation. When the length of the CRL is greater than the threshold, all unrevoked vehicles in vehicular networks are required to update their public keys and private keys. In our scheme, we reduce the length of the CRL which will lower the overhead of key updates.

\section{CONCLUSIONS}

In this paper, we proposed a flexible and efficient delayaware authentication scheme (FEDAS) for vehicular networks utilizing the edge computing paradigm. By introducing local CRL and decreasing the length of CRL that is used in the verification process, we showed that our proposed FEDAS reduced the signature verification delay. We introduced transition zone and corresponding operations to deal with the conflict of authentication in the boarder of the sub-area. We also showed the strong security property of FEDAS in the aspect of 
entity authentication, data integrity, anonymity, unlinkability, traceability, revocation and three types of attacks.

We implemented the proposed authentication architecture by further proposing an area division method that minimizes the number of groups in the entire network. The main consideration with the proposed authentication is the level of granularity of area partitioning such that the size of each group is set adequately to ensure vehicles do not experience excessive delay in processing security related operations, while the number of groups in the entire network can be kept low. As area partitioning depends on traffic volumes in an area, we developed an analytical model to translate high level statistics of traffic volumes to the length of local CRL which directly influences the delay performance. We then conducted intensive simulation experiments to test the performance of our proposed FEDAS showing the effect of area division on the delay performance.

\section{REFERENCES}

[1] 3GPP TR 22.886 v16.1.1, "Study on enhancement of 3GPP support for 5G V2X services," Tech. Rep., Sep. 2018.

[2] M. Amoozadeh, A. Raghuramu, C. Chuah, D. Ghosal, H. M. Zhang, J. Rowe, and K. Levitt, "Security vulnerabilities of connected vehicle streams and their impact on cooperative driving," IEEE Communications Magazine, vol. 53, no. 6, pp. 126-132, Jun. 2015.

[3] P. Asuquo, H. Cruickshank, J. Morley, C. P. A. Ogah, A. Lei, W. Hathal, S. Bao, and Z. Sun, "Security and privacy in location-based services for vehicular and mobile communications: An overview, challenges and countermeasures," IEEE Internet of Things Journal, pp. 1-25, 2018.

[4] M. Raya and J.-P. Hubaux, "Securing vehicular ad hoc networks," Journal of Computer Security, vol. 15, no. 1, pp. 39-68, Jan. 2007.

[5] F. Qu, Z. Wu, F. Wang, and W. Cho, "A security and privacy review of VANETs," IEEE Transactions on Intelligent Transportation Systems, vol. 16, no. 6, pp. 2985-2996, Dec. 2015.

[6] J. Ni, A. Zhang, X. Lin, and X. S. Shen, "Security, privacy, and fairness in fog-based vehicular crowdsensing," IEEE Communications Magazine, vol. 55, no. 6, pp. 146-152, Jun. 2017.

[7] M. Azees, P. Vijayakumar, and L. J. Deboarh, "EAAP: Efficient anonymous authentication with conditional privacy-preserving scheme for vehicular ad hoc networks," IEEE Transactions on Intelligent Transportation Systems, vol. 18, no. 9, pp. 2467-2476, Sep. 2017.

[8] J. Li, H. Lu, and M. Guizani, "ACPN: A novel authentication framework with conditional privacy-preservation and non-repudiation for VANETs," IEEE Transactions on Parallel and Distributed Systems, vol. 26, no. 4, pp. 938-948, Apr. 2015.

[9] D. Chaum and E. van Heyst, "Group signatures," in Advances in Cryptology - EUROCRYPT '91. Berlin, Heidelberg: Springer Berlin Heidelberg, 1991, pp. 257-265.

[10] M. Bellare, D. Micciancio, and B. Warinschi, "Foundations of group signatures: Formal definitions, simplified requirements, and a construction based on general assumptions," in Advances in Cryptology - EUROCRYPT 2003. Berlin, Heidelberg: Springer Berlin Heidelberg, 2003, pp. 614-629.

[11] M. Manulis, N. Fleischhacker, F. Gnther, F. Kiefer, and B. Poettrering. (2012) Group signatures: Authentication with privacy. Bundesamt fur Sicherheit in der Informationstechnik. Bonn, Germany. [Online]. Available: https://www.bsi.bund.de/DE/Publikationen/Studien/ GroupSignatures/GruPA.html

[12] D. Cooper and S. Santesson and S. Farrell and S. Boeyen and R. Housley and W. Polk, "Internet X.509 Public Key Infrastructure Certificate and Certificate Revocation List (CRL) Profile," RFC 5280, May 2008.

[13] D. Boneh and H. Shacham, "Group signatures with verifier-local revocation," in Proceedings of the 11th ACM Conference on Computer and Communications Security, 2004, pp. 168-177.

[14] M. Satyanarayanan, "The emergence of edge computing," Computer, vol. 50, no. 1, pp. 30-39, Jan 2017.

[15] Y. Mao, C. You, J. Zhang, K. Huang, and K. B. Letaief, "A survey on mobile edge computing: The communication perspective," IEEE Cотmunications Surveys Tutorials, vol. 19, no. 4, pp. 2322-2358, Fourthquarter 2017.
[16] T. Taleb, K. Samdanis, B. Mada, H. Flinck, S. Dutta, and D. Sabella, "On multi-access edge computing: A survey of the emerging $5 \mathrm{~g}$ network edge cloud architecture and orchestration," IEEE Communications Surveys Tutorials, vol. 19, no. 3, pp. 1657-1681, Thirdquarter 2017.

[17] Z. Lu, G. Qu, and Z. Liu, "A survey on recent advances in vehicular network security, trust, and privacy," IEEE Transactions on Intelligent Transportation Systems, vol. 20, no. 2, pp. 760-776, Feb 2019.

[18] J. Petit, F. Schaub, M. Feiri, and F. Kargl, "Pseudonym schemes in vehicular networks: A survey," IEEE Communications Surveys Tutorials, vol. 17, no. 1, pp. 228-255, Firstquarter 2015.

[19] A. Boualouache, S. Senouci, and S. Moussaoui, "A survey on pseudonym changing strategies for vehicular ad-hoc networks," IEEE Communications Surveys Tutorials, vol. 20, no. 1, pp. 770-790, Firstquarter 2018.

[20] D. Forster, H. Lohr, A. Gratz, J. Petit, and F. Kargl, "An evaluation of pseudonym changes for vehicular networks in large-scale, realistic traffic scenarios," IEEE Transactions on Intelligent Transportation Systems, vol. 19, no. 10, pp. 3400-3405, 2018.

[21] D. Boneh, X. Boyen, and H. Shacham, "Short group signatures," in Advances in Cryptology - CRYPTO 2004, M. Franklin, Ed., Berlin, Heidelberg, 2004, pp. 41-55.

[22] L. Zhang, C. Li, Y. Li, Q. Luo, and R. Zhu, "Group signature based privacy protection algorithm for mobile ad hoc network," in 2017 IEEE International Conference on Information and Automation (ICIA), Jul. 2017, pp. 947-952.

[23] A. Wasef and X. Shen, "Efficient group signature scheme supporting batch verification for securing vehicular networks," in 2010 IEEE International Conference on Communications, May 2010, pp. 1-5.

[24] S. Jiang, X. Zhu, and L. Wang, "An efficient anonymous batch authentication scheme based on HMAC for VANETs," IEEE Transactions on Intelligent Transportation Systems, vol. 17, no. 8, pp. 2193-2204, Aug. 2016.

[25] L. Zhang, Q. Wu, A. Solanas, and J. Domingo-Ferrer, "A scalable robust authentication protocol for secure vehicular communications," IEEE Transactions on Vehicular Technology, vol. 59, no. 4, pp. 16061617, May 2010.

[26] J. Shao, X. Lin, R. Lu, and C. Zuo, "A threshold anonymous authentication protocol for VANETs," IEEE Transactions on Vehicular Technology, vol. 65 , no. 3, pp. 1711-1720, Mar. 2016.

[27] X. Yue, B. Chen, X. Wang, Y. Duan, M. Gao, and Y. He, "An efficient and secure anonymous authentication scheme for VANETs based on the framework of group signatures," IEEE Access, vol. 6, pp. 62 584-62 600, 2018.

[28] A. Lei, H. Cruickshank, Y. Cao, P. Asuquo, C. P. A. Ogah, and Z. Sun, "Blockchain-based dynamic key management for heterogeneous intelligent transportation systems," IEEE Internet of Things Journal, vol. 4, no. 6, pp. 1832-1843, 2017.

[29] S. A. George, A. Jaekel, and I. Saini, "Secure identity management framework for vehicular ad-hoc network using blockchain," in 2020 IEEE Symposium on Computers and Communications (ISCC), 2020, pp. 1-6.

[30] A. Dorri, M. Steger, S. S. Kanhere, and R. Jurdak, "Blockchain: A distributed solution to automotive security and privacy," IEEE Communications Magazine, vol. 55, no. 12, pp. 119-125, 2017.

[31] Z. Ma, J. Zhang, Y. Guo, Y. Liu, X. Liu, and W. He, "An efficient decentralized key management mechanism for vanet with blockchain," IEEE Transactions on Vehicular Technology, vol. 69, no. 6, pp. 58365849,2020

[32] Q. Feng, D. He, S. Zeadally, and K. Liang, "BPAS: Blockchain-assisted privacy-preserving authentication system for vehicular ad hoc networks," IEEE Transactions on Industrial Informatics, vol. 16, no. 6, pp. 41464155,2020

[33] W. Li, H. Guo, M. Nejad, and C. C. Shen, "Privacy-preserving traffic management: A blockchain and zero-knowledge proof inspired approach," IEEE Access, vol. 8, pp. 181 733-181 743, 2020.

[34] Z. Yang, K. Yang, L. Lei, K. Zheng, and V. C. M. Leung, "Blockchainbased decentralized trust management in vehicular networks," IEEE Internet of Things Journal, vol. 6, no. 2, pp. 1495-1505, 2019.

[35] X. Chen, J. Ding, and Z. Lu, "A decentralized trust management system for intelligent transportation environments," IEEE Transactions on Intelligent Transportation Systems, vol. Early Access, pp. 1-14, 2020.

[36] M. Saad, J. Spaulding, L. Njilla, C. Kamhoua, S. Shetty, D. Nyang, and D. Mohaisen, "Exploring the attack surface of blockchain: A comprehensive survey," IEEE Communications Surveys Tutorials, vol. 22, no. 3, pp. 1977-2008, Thirdquarter 2020.

[37] A. M. Antonopoulos, Mastering Bitcoin: Programming the Open Blockchain, 2nd ed. OReilly Media, 2017. 
[38] L. Chen, S. Ng, and G. Wang, "Threshold anonymous announcement in VANETs," IEEE Journal on Selected Areas in Communications, vol. 29, no. 3, pp. 605-615, Mar. 2011.

[39] X. Lin, X. Sun, P. Ho, and X. Shen, "GSIS: A secure and privacypreserving protocol for vehicular communications," IEEE Transactions on Vehicular Technology, vol. 56, no. 6, pp. 3442-3456, Nov. 2007.

[40] MEC, the Multi-access Edge Computing. [Online]. Available: https://www.etsi.org/technologies/multi-access-edge-computing

[41] R. Diestel, Graph Theory, 2nd ed. Springer-Verlag New York, 2000.

[42] A. Kumar, D. Manjunath, and J. Kuri, Communication networking: an analytical approach. Elsevier, 2004.

[43] The Annual Report for Transport of Beijing (2019). [Online]. Available: http://www.bjtrc.org.cn/List/index/cid/7.html

[44] SUMO, Simulation of Urban MObility. [Online]. Available: http://sumo.sourceforge.net

[45] NS3, Network Simulator version 3. [Online]. Available: https://www.nsnam.org

[46] PBC, the Pairing-Based Cryptography library. [Online]. Available: https://crypto.stanford.edu/pbc/

[47] A. Miyaji, M. Nakabayashi, and S. Takano, "New explicit conditions of elliptic curve traces for FR-reduction," IEICE transactions on fundamentals of electronics, communications and computer sciences, vol. E84-A, no. 5, pp. 1234-1243, 2001.

[48] GCC, the GNU Compiler Collection. [Online]. Available: http://gcc.gnu.org/ 